ISSN 2078-3744. Вісник Львів. ун-ту. Серія мех.-мат. 2020. Випуск 89. С. 39-59

Visnyk of the Lviv Univ. Series Mech. Math. 2020. Issue 89. P. 39-59

http://publications.lnu.edu.ua/bulletins/index.php/mmf

doi: http://dx.doi.org/10.30970/vmm.2020.89.039-059

УДК 515.12

\title{
HOMEOMORPHISMS OF THE SPACE OF NONZERO INTEGERS WITH THE KIRCH TOPOLOGY
}

\author{
Yaryna STELMAKH \\ Ivan Franko National University of Lviv, \\ Universitetska Str., 1, 79000, Lviv, Ukraine \\ e-mails: yarynziya@ukr.net
}

The Golomb (resp. Kirch) topology on the set $\mathbb{Z}^{\bullet}$ of nonzero integers is generated by the base consisting of arithmetic progressions $a+b \mathbb{Z}=\{a+b n$ : $n \in \mathbb{Z}\}$ where $a \in \mathbb{Z}^{\bullet}$ and $b$ is a (square-free) number, coprime with $a$. In 2019 Dario Spirito proved that the space of nonzero integers endowed with the Golomb topology admits only two self-homeomorphisms. In this paper we prove an analogous fact for the space of nonzero integers endowed with the Kirch topology: it also admits exactly two self-homeomorphisms.

Key words: Kirch topology, superconnected space, superconnecting poset.

In this paper we describe the homeomorphism group of the space $\mathbb{Z} \bullet$ of nonzero integers endowed with the Kirch topology $\tau_{K}$, which is generated by the subbase consisting of the cosets $a+p \mathbb{Z}$ where $a \in \mathbb{Z} \bullet$ and $p$ is a prime number that does not divide $a$. On the subspace $\mathbb{N}$ of $\mathbb{Z} \bullet$ this topology was introduced by Kirch in [6.

Banakh, Stelmakh and Turek [3] proved that the subspace $\mathbb{N}$ of $\left(\mathbb{Z} \bullet, \tau_{K}\right)$ is topologically rigid in the sense that each self-homeomorphism of $\mathbb{N}$ endowed with the subspace topology $\tau_{K} \mid \mathbb{N}=\{U \cap \mathbb{N}: U \in \tau\}$ is the identity map of $\mathbb{N}$.

On the other hand, the space $\left(\mathbb{Z}^{\bullet}, \tau_{K}\right)$ does admit a non-trivial self-homeomorpfism, namely the map

$$
j: \mathbb{Z}^{\bullet} \rightarrow \mathbb{Z}^{\bullet}, \quad j: x \mapsto-x .
$$

In this paper we prove that this is the unique non-trivial self-homeomorphism of the topological space $\left(\mathbb{Z}^{\bullet}, \tau_{K}\right)$. A similar result for the Golomb topology on $\mathbb{Z}^{\bullet}$ was proved by Dario Spirito [11. The topological rigidity of the Golomb topology on $\mathbb{N}$ was proved by Banakh, Spirito and Turek in [2].

Theorem 1. The space $\mathbb{Z} \bullet=\mathbb{Z} \backslash\{0\}$ of nonzero integers endowed with the Kirch topology admits only two self-homeomorphisms.

2020 Mathematics Subject Classification: 54D05, 54H99, 11A41, 11N13.

(C) Stelmakh, Ya., 2020 
The proof of this theorem follows the lines of the proof of the topological rigidity of the space $\left(\mathbb{N}, \tau_{K} \backslash \mathbb{N}\right)$ from [3]. The proof is divided into 23 lemmas. A crucial role in the proof belongs to the superconnectedness of the Kirch space and the superconnecting poset of the Kirch space, which is defined in Section 2 .

\section{Four Classical NUMBER-Theoretic RESUlts}

By $\Pi$ we denote the set of prime numbers. For a number $x \in \mathbb{Z}$ by $\Pi_{x}$ we denote the set of all prime divisors of $x$. Two numbers $x, y \in \mathbb{Z}$ are coprime iff $\Pi_{x} \cap \Pi_{y}=\varnothing$.

In the proof of Theorem 1 we shall exploit the following four known results of Number Theory. The first one is the famous Chinese Remainder Theorem (see. e.g. [5, $3.12])$.

Theorem 2 (Chinese Remainder Theorem). If $b_{1}, \ldots, b_{n} \in \mathbb{Z}$ are pairwise coprime numbers, then for any numbers $a_{1}, \ldots, a_{n} \in \mathbb{Z}$, the intersection $\bigcap_{i=1}^{n}\left(a_{i}+b_{i} \mathbb{N}\right)$ is infinite.

The second classical result is not elementary and is due to Dirichlet [4, S.VI], see also [1, Ch.7].

Theorem 3 (Dirichlet). For any coprime numbers $a, b \in \mathbb{N}$ the arithmetic progression $a+b \mathbb{N}$ contains a prime number.

The third classical result is a recent theorem of Mihallescu [8] who solved old Catalan's Conjecture [7].

Theorem 4 (Mihăilescu). If $a, b \in\left\{m^{n+1}: n, m \in \mathbb{N}\right\}$, then $|a-b|=1$ if and only if $\{a, b\}=\left\{2^{3}, 3^{2}\right\}$.

The fourth classical result we use is due to Karl Zsigmondy [12, see also [10, Theorem 3].

Theorem 5 (Zsigmondy). For integer numbers $a, n \in \mathbb{N} \backslash\{1\}$ the inclusion

$$
\Pi_{a^{n}-1} \subseteq \bigcup_{0<k<n} \Pi_{a^{k}-1}
$$

holds if and only if one of the following conditions is satisfied:

(1) $n=2$ and $a=2^{k}-1$ for some $k \in \mathbb{N}$; then

$$
a^{2}-1=(a+1)(a-1)=2^{k}(a-1) ;
$$

(2) $n=6$ and $a=2$; then

$$
a^{n}-1=2^{6}-1=63=3^{2} \times 7=\left(a^{2}-1\right)^{2} \times\left(a^{3}-1\right) .
$$

\section{SUPERCONNECTED SPACES AND THEIR SUPERCONNECTING POSETS}

In this section we discuss superconnected topological spaces and some order structures related to such spaces.

First let us introduce some notation and recall some notions.

For a set $A$ and $n \in \omega$ let $[A]^{n}=\{E \subseteq A:|A|=n\}$ be the family of $n$-element subsets of $A$, and $[A]^{<\omega}=\bigcup_{n \in \omega}[A]^{n}$ be the family of all finite subsets of $A$. For a function 
$f: X \rightarrow Y$ and a subset $A \subseteq X$ by $f[A]$ we denote the image $\{f(a): a \in A\}$ of the set $A$ under the function $f$.

For a subset $A$ of a topological space $(X, \tau)$ by $\bar{A}$ we denote the closure of $A$ in $X$. For a point $x \in X$ we denote by $\tau_{x}:=\{U \in \tau: x \in U\}$ the family of all open neighborhoods of $x$ in $(X, \tau)$. A poset is an abbreviation for a partially ordered set.

A family $\mathcal{F}$ of subsets of a set $X$ is called a filter if

- $\varnothing \notin \mathcal{F}$;

- for any $A, B \in \mathcal{F}$ their intersection $A \cap B \in \mathcal{F}$;

- for any sets $F \subseteq E \subseteq X$ the inclusion $F \in \mathcal{F}$ implies $E \in \mathcal{F}$.

A topological space $(X, \tau)$ is called superconnected if for any $n \in \mathbb{N}$ and non-empty open sets $U_{1}, \ldots, U_{n}$ the intersection $\overline{U_{1}} \cap \cdots \cap \overline{U_{n}}$ is non-empty. This allows us to define the filter

$$
\mathcal{F}_{\infty}=\left\{B \subseteq X: \exists U_{1}, \ldots, U_{n} \in \tau \backslash\{\varnothing\} \quad\left(\overline{U_{1}} \cap \cdots \cap \overline{U_{n}} \subseteq B\right)\right\}
$$

called the superconnecting filter of $X$.

For every finite subset $E$ of $X$ consider the subfilter

$$
\mathcal{F}_{E}:=\left\{B \subseteq X: \exists\left(U_{x}\right)_{x \in E} \in \prod_{x \in E} \tau_{x}\left(\bigcap_{x \in E} \overline{U_{x}} \subseteq B\right)\right\}
$$

of $\mathcal{F}_{\infty}$. Here we assume that $\mathcal{F}_{\varnothing}=\{X\}$. It is clear that for any finite sets $E \subseteq F$ in $X$ we have $\mathcal{F}_{E} \subseteq \mathcal{F}_{F}$.

The family

$$
\mathfrak{F}=\left\{\mathcal{F}_{E}: E \in[X]^{<\omega}\right\} \cup\left\{\mathcal{F}_{\infty}\right\}
$$

is endowed with the inclusion partial order and is called the superconnecting poset of the superconnected space $X$. The filters $\mathcal{F}_{\varnothing}$ and $\mathcal{F}_{\infty}$ are the smallest and largest elements of the poset $\mathfrak{F}$, respectively.

The following obvious lemma shows that the superconnecting poset $\mathfrak{F}$ is a topological invariant of the superconnected space.

Proposition 1. For any homeomorphism $h$ of any superconnected topological space $X$, the map

$$
\tilde{h}: \mathfrak{F} \rightarrow \mathfrak{F}, \quad \tilde{h}: \mathcal{F} \mapsto\{h[A]: A \in \mathcal{F}\},
$$

is an order isomorphism of the superconnecting poset $\mathfrak{F}$.

In the following sections we shall study the order properties of the poset $\mathfrak{F}$ for the Kirch space $\left(\mathbb{Z}^{\bullet}, \tau_{K}\right)$ and shall exploit the obtained information in the proof of the topological rigidity of the Kirch space.

\section{Proof of Theorem 1}

We divide the proof of Theorem 1 into 23 lemmas.

Lemma 1. For any $a, b \in \mathbb{Z} \bullet$ the closure $\overline{a+b \mathbb{Z}}$ of the arithmetic progression $a+b \mathbb{Z}$ in the Kirch space $\left(\mathbb{Z} \bullet, \tau_{K}\right)$ is equal to

$$
\mathbb{Z} \bullet \cap \bigcap_{p \in \Pi_{b}}(\{0, a\}+p \mathbb{Z}) .
$$


Proof. First we prove that $\overline{a+b \mathbb{Z}} \subseteq\{0, a\}+p \mathbb{Z}$ for every $p \in \Pi_{b}$. Take any point $x \in \overline{a+b \mathbb{Z}}$ and assume that $x \notin p \mathbb{Z}$. Then $x+p \mathbb{Z}$ is a neighborhood of $x$ and hence the intersection $(x+p \mathbb{Z}) \cap(a+b \mathbb{Z})$ is not empty. Then there exist $u, v \in \mathbb{Z}$ such that $x+p u=a+b v$. Consequently, $x-a=b v-p u \in p \mathbb{Z}$ and $x \in a+p \mathbb{Z}$.

Next, take any point $x \in \mathbb{Z} \bullet \cap \bigcap_{p \in \Pi_{b}}(\{0, a\}+p \mathbb{Z})$. Given any neighborhood $O_{x}$ of $x$ in $\left(\mathbb{Z}^{\bullet}, \tau_{K}\right)$, we should prove that $O_{x} \cap(a+b \mathbb{Z}) \neq \varnothing$. By the definition of the Kirch topology there exists a square-free number $d \in \mathbb{Z} \bullet$ such that $d, x$ are coprime and $x+d \mathbb{Z} \subseteq O_{x}$.

If $\Pi_{b} \subseteq \Pi_{x}$, then $b, d$ are coprime and by the Chinese Remainder Theorem

$$
\varnothing \neq(x+d \mathbb{Z}) \cap(a+b \mathbb{Z}) \subseteq O_{x} \cap(a+b \mathbb{Z}) .
$$

So, we can assume $\Pi_{b} \backslash \Pi_{x} \neq \varnothing$. The choice of $x \in \bigcap_{p \in \Pi_{b}}(\{0, a\}+p \mathbb{Z})$ guarantees that

$$
x \in \bigcap_{p \in \Pi_{b} \backslash \Pi_{x}}(a+p \mathbb{Z})=a+q \mathbb{Z}
$$

where $q=\prod_{p \in \Pi_{b} \backslash \Pi_{x}} p$. Since the numbers $x$ and $d$ are coprime and $d$ is square-free, the greatest common divisor of $b$ and $d$ divides the number $q$. Since $x-a \in q \mathbb{Z}$, the Euclides algorithm yields two numbers $u, v \in \mathbb{Z}$ such that $x-a=b u-d v$, which implies that $O_{x} \cap(a+b \mathbb{Z}) \supseteq(x+d \mathbb{Z}) \cap(a+b \mathbb{Z}) \neq \varnothing$.

Lemma 1 implies that the Kirch space $\left(\mathbb{Z}^{\bullet}, \tau_{K}\right)$ is superconnected and hence possesses the superconnecting filter

$$
\mathcal{F}_{\infty}=\left\{F \subseteq \mathbb{Z}^{\bullet}: \exists U_{1}, \ldots, U_{n} \in \tau_{K} \backslash\{\varnothing\} \quad\left(\bigcap_{i=1}^{n} \overline{U_{i}} \subseteq F\right)\right\}
$$

and the superconnecting poset

$$
\mathfrak{F}=\left\{\mathcal{F}_{E}: E \in\left[\mathbb{Z}^{\bullet}\right]^{<\omega}\right\} \cup\left\{\mathcal{F}_{\infty}\right\}
$$

consisting of the filters

$$
\mathcal{F}_{E}=\left\{F \subseteq \mathbb{Z} \bullet: \exists\left(U_{x}\right)_{x \in E} \in \prod_{x \in E} \tau_{x}\left(\bigcap_{x \in E} \overline{U_{x}} \subseteq F\right)\right\} .
$$

Here for a point $x \in \mathbb{Z}^{\bullet}$ by $\tau_{x}:=\left\{U \subseteq \mathbb{Z}^{\bullet}: x \in U\right\}$ we denote the family of open neighborhoods of $x$ in the Kirch topology $\tau_{K}$.

For a nonempty finite subset $E \subseteq \mathbb{Z}^{\bullet}$, let $\Pi_{E}=\bigcap_{x \in E} \Pi_{x}$ be the set of common prime divisors of numbers in the set $E$. Also let

$$
A_{E}=\{p \in \Pi: \exists k \in \mathbb{N}(E \subset\{0, k\}+p \mathbb{Z})\} .
$$

Observe that $\Pi_{E} \subseteq A_{E}$ and $A_{E} \neq \varnothing$ because $2 \in A_{E}$. If $E$ is a singleton, then $A_{E}=\Pi$; if $|E| \geq 2$, then

$$
A_{E} \subseteq \Pi_{x} \cup \Pi_{y} \cup \Pi_{x-y} \subseteq\{2, \ldots, \max E\}
$$

for any distinct numbers $x, y \in E$. This inclusion follows from

Lemma 2. For any two-element set $E=\{x, y\} \subset \mathbb{Z} \bullet$ we have $A_{E}=\Pi_{x} \cup \Pi_{y} \cup \Pi_{x-y}$. 
Proof. Each number $p \in \Pi_{x}$ (resp. $p \in \Pi_{y}$ ) belongs to $A_{E}$ because $\{x, y\} \subset\{0, y\}+p \mathbb{Z}$ (resp. $\{x, y\} \subset\{0, x\}+p \mathbb{Z}\}$ ). Each number $p \in \Pi_{x-y}$ belongs to $A_{E}$ because $\{x, y\} \subset$ $x+p \mathbb{Z} \subseteq\{0, x\}+p \mathbb{Z}$. This proves that $\Pi_{x} \cup \Pi_{y} \cup \Pi_{x-y} \subseteq A_{E}$.

Now take any prime number $p \in A_{E}$ and assume that $p \notin \Pi_{x} \cup \Pi_{y}$. It follows from $\{x, y\}=E \subset\left\{0, \alpha_{E}(p)\right\}+p \mathbb{Z}$ that $\{x, y\} \subseteq \alpha_{E}(p)+p \mathbb{Z}$ and hence $x-y \in p \mathbb{Z}$ and $p \in \Pi_{x-y}$.

Let $\alpha_{E}: A_{E} \rightarrow \omega$ be the unique function satisfying the following conditions:

(i) $\alpha_{E}(p)<p$ for all $p \in A_{E}$;

(ii) $E \subseteq\left\{0, \alpha_{E}(p)\right\}+p \mathbb{Z}$ for all $p \in A_{E}$;

(iii) $\alpha_{E}(2)=1$ and $\alpha_{E}(p)=0$ for all $p \in \Pi_{E} \backslash\{2\}$.

Lemma 3. Let $A \subset \Pi$ be a finite set containing 2 and $\alpha: A \rightarrow \mathbb{N}_{0}$ be a function such that $\alpha(2)=1$ and $\alpha(p) \in\{0, \ldots, p-1\}$ for all $p \in A \backslash\{2\}$. Let $x$ be the product of odd prime numbers in the set $A$ and $y$ be any number in the set $\mathbb{Z} \bullet \cap \bigcap_{p \in A}(\alpha(p)+p \mathbb{Z})$. Then the set $E=\{y, x, 2 x\}$ has $A_{E}=A$ and $\alpha_{E}=\alpha$.

Proof. For every prime number $p \in A$ we have $\{x, y\} \subset\{0, y\}+p \mathbb{Z}$, which implies that $p \in A_{E}$. Assuming that $A_{E} \backslash A$ contains some prime number $p$, we conclude that $x \notin p \mathbb{Z}$ and hence the inclusion $\{y, x, 2 x\}=E \subset\left\{0, \alpha_{E}(p)\right\}+p \mathbb{Z}$ implies $\{x, 2 x\} \subset \alpha_{E}(p)+p \mathbb{Z}$ and $x=2 x-x \in p \mathbb{Z}$. This contradiction shows that $A_{E}=A$. To show that $\alpha_{E}=\alpha$, take any prime number $p \in A=A_{E}$. If $p=2$, then $\alpha(p)=1=\alpha_{E}(p)$. So, we assume that $p \neq 2$. If $\alpha(p)=0$, then $y \in \alpha(p)+p \mathbb{Z}=p \mathbb{Z}$ and hence $p \in \Pi_{E}$. In this case $\alpha_{E}(p)=0=\alpha(p)$. If $\alpha(p) \neq 0$, then the number $y \in \alpha(p)+p \mathbb{Z}$ is not divisible by $p$ and then the inclusions $\{y, x, 2 x\} \subseteq\{0, \alpha(p)\}+p \mathbb{Z}$ and $\{y, x, 2 x\}=E \subset\left\{0, \alpha_{E}(p)\right\}+p \mathbb{Z}$ imply that $\alpha(p)=\alpha_{E}(p)$.

The following lemma yields an arithmetic description of the filters $\mathcal{F}_{E}$.

Lemma 4. For any finite subset $E \subseteq \mathbb{Z} \bullet$ with $|E| \geq 2$ we have

$$
\mathcal{F}_{E}=\left\{B \subseteq \mathbb{Z}^{\bullet}: \exists L \in\left[\Pi \backslash A_{E}\right]^{<\omega} \bigcap_{p \in L} p \mathbb{Z}^{\bullet} \cap \bigcap_{p \in A_{E} \backslash \Pi_{E}}\left(\left\{0, \alpha_{E}(p)\right\}+p \mathbb{Z}\right) \subseteq B\right\} .
$$

Here we assume that $\bigcap_{p \in \varnothing} p \mathbb{Z}^{\bullet}=\mathbb{Z}^{\bullet}$.

Proof. It suffices to verify two properties:

(1) for any $\left(U_{x}\right)_{x \in E} \in \prod_{x \in E} \tau_{x}$ there exists a finite set $L \subseteq \Pi \backslash A_{E}$ such that

$$
\bigcap_{p \in L} p \mathbb{Z} \bullet \cap \bigcap_{p \in A_{E} \backslash \Pi_{E}}\left(\left\{0, \alpha_{E}(p)\right\}+p \mathbb{Z}\right) \subseteq \bigcap_{x \in E} \overline{U_{x}}
$$

(2) for any finite set $L \subseteq \Pi \backslash A_{E}$ there exists a sequence of neighborhoods $\left(U_{x}\right)_{x \in E} \in$ $\prod_{x \in E} \tau_{x}$ such that

$$
\bigcap_{x \in E} \overline{U_{x}} \subseteq \bigcap_{p \in L} p \mathbb{Z}^{\bullet} \cap \bigcap_{p \in A_{E} \backslash \Pi_{E}}\left(\left\{0, \alpha_{E}(p)\right\}+p \mathbb{Z}\right)
$$


1. Given a sequence of neighborhoods $\left(U_{x}\right)_{x \in E} \in \prod_{x \in E} \tau_{x}$, for every $x \in E$ find a square-free number $q_{x}>x$ such that $\Pi_{q_{x}} \cap \Pi_{x}=\varnothing$ and $x+q_{x} \mathbb{Z} \subseteq U_{x}$. We claim that the finite set $L=\bigcup_{x \in E} \Pi_{q_{x}} \backslash A_{E}$ has the required property. Given any number

$$
z \in \bigcap_{p \in L} p \mathbb{Z}^{\bullet} \cap \bigcap_{p \in A_{E} \backslash \Pi_{E}}\left(\left\{0, \alpha_{E}(p)\right\}+p \mathbb{Z}\right),
$$

we should prove that $z \in \overline{U_{x}}$ for every $x \in E$. By Lemma 1

$$
\mathbb{Z} \bullet \bigcap_{p \in \Pi_{q_{x}}}(\{0, x\}+p \mathbb{Z})=\overline{\left(x+q_{x} \mathbb{Z}\right)} \subseteq \overline{U_{x}} .
$$

So, it suffices to show that $z \in\{0, x\}+p \mathbb{Z}$ for any $p \in \Pi_{q_{x}}$. Since the numbers $x$ and $q_{x}$ are coprime, $p \notin \Pi_{x}$ and hence $p \notin \Pi_{E}$. If $p \notin A_{E}$, then $p \in \Pi_{q_{x}} \backslash A_{E} \subseteq L$ and hence $z \in p \mathbb{N} \subseteq\{0, x\}+p \mathbb{Z}$. If $p \in A_{E}$, then $x \in E \subseteq\left\{0, \alpha_{E}(p)\right\}+p \mathbb{Z}$ and $x \in \alpha_{E}(p)+p \mathbb{Z}$ (as $\left.p \notin \Pi_{x}\right)$. Then $x+p \mathbb{Z}=\alpha_{E}(p)+p \mathbb{Z}$ and $z \in\left\{0, \alpha_{E}(p)\right\}+p \mathbb{Z}=\{0, x\}+p \mathbb{Z}$.

2. Fix any finite set $L \subseteq \Pi \backslash A_{E}$. For every $x \in E$ consider the neighborhood $U_{x}=\bigcap_{p \in L \cup A_{E} \backslash \Pi_{x}}(x+p \mathbb{Z})$ of $x$ in the Kirch topology. By Lemma 1 ,

$$
\overline{U_{x}}=\mathbb{Z}^{\bullet} \cap \bigcap_{p \in L \cup A_{E} \backslash \Pi_{x}}(\{0, x\}+p \mathbb{Z}) .
$$

Given any number $z \in \bigcap_{x \in E} \overline{U_{x}}$, we should show that

$$
z \in \bigcap_{p \in L} p \mathbb{Z}^{\bullet} \cap \bigcap_{p \in A_{E} \backslash \Pi_{E}}\left(\left\{0, \alpha_{E}(p)\right\}+p \mathbb{Z}\right) .
$$

This will follow as soon as we check that $z \in p \mathbb{Z} \bullet$ for all $p \in L$ and $z \in\left\{0, \alpha_{E}(p)\right\}+p \mathbb{Z}$ for all $p \in A_{E} \backslash \Pi_{E}$.

Given any $p \in A_{E} \backslash \Pi_{E}$, we can find a point $x \in E \backslash p \mathbb{Z}$ and observe that $x \in E \subseteq$ $\left\{0, \alpha_{E}(p)\right\}+p \mathbb{Z}$. Then

$$
z \in \overline{U_{x}} \subseteq \overline{x+p \mathbb{Z}} \subseteq\{0, x\}+p \mathbb{Z}=\left\{0, \alpha_{E}(p)\right\}+p \mathbb{Z} .
$$

Now take any prime number $p \in L$. Since $L \cap A_{E}=\varnothing$, we conclude that $E \nsubseteq p \mathbb{Z}$. So, we can fix a number $x \in E \backslash p \mathbb{Z}$. Taking into account that $p \notin A_{E}$, we conclude that $E \nsubseteq\{0, x\}+p \mathbb{Z}$ and hence there exists a number $y \in E$ such that $p \mathbb{Z} \neq y+p \mathbb{Z} \neq x+p \mathbb{Z}$. Then

$$
z \in \overline{U_{x}} \cap \overline{U_{y}} \subseteq(\{0, x\}+p \mathbb{Z}) \cap(\{0, y\}+p \mathbb{Z})=p \mathbb{Z}
$$

We shall use Lemma 4 for an arithmetic characterization of the partial order of the superconnecting poset $\mathfrak{F}$ of the Kirch space.

Lemma 5. For two finite subsets $E, F \subseteq \Pi$ with $\min \{|E|,|F|\} \geq 2$ we have $\mathcal{F}_{E} \subseteq \mathcal{F}_{F}$ if and only if

$$
A_{F} \subseteq A_{E}, \quad \Pi_{F} \backslash\{2\} \subseteq \Pi_{E} \quad \text { and } \quad \alpha_{E}\left\lceil A_{F} \backslash \Pi_{E}=\alpha_{F}\left\lceil A_{F} \backslash \Pi_{E} .\right.\right.
$$


Proof. To prove the "only if" part, assume that $\mathcal{F}_{E} \subseteq \mathcal{F}_{F}$. By Lemma 4 the set

$$
\bigcap_{p \in A_{F} \backslash A_{E}} p \mathbb{Z} \bullet \bigcap_{p \in A_{E} \backslash \Pi_{E}}\left(\left\{0, \alpha_{E}(p)\right\}+p \mathbb{Z}\right)
$$

belongs to the filter $\mathcal{F}_{E} \subseteq \mathcal{F}_{F}$. By Lemma 4 , there exists a finite set $L \subset \Pi \backslash A_{F}$ such that

$$
\bigcap_{p \in L} p \mathbb{Z}^{\bullet} \cap \bigcap_{p \in A_{F} \backslash \Pi_{F}}\left(\left\{0, \alpha_{F}(p)\right\}+p \mathbb{Z}\right) \subseteq \bigcap_{p \in A_{F} \backslash A_{E}} p \mathbb{Z}^{\bullet} \cap \bigcap_{p \in A_{E} \backslash \Pi_{E}}\left(\left\{0, \alpha_{E}(p)\right\}+p \mathbb{Z}\right) .
$$

This inclusion combined with the Chinese Remainder Theorem 2 implies

$$
A_{F} \backslash A_{E} \subseteq L \subset \Pi \backslash A_{F}, \quad A_{E} \backslash\left(\Pi_{E} \cup\{2\}\right) \subseteq L \cup\left(A_{F} \backslash \Pi_{F}\right)
$$

and

and

$$
\alpha_{E}(p)=\alpha_{F}(p) \quad \text { for any } \quad p \in\left(A_{F} \backslash \Pi_{F}\right) \cap\left(A_{E} \backslash \Pi_{E}\right),
$$

$$
A_{F} \subseteq A_{E}, \quad \Pi_{F} \backslash\{2\} \subseteq \Pi_{E} \quad \text { and } \quad \alpha_{E}\left\lceil A_{F} \backslash \Pi_{E}=\alpha_{F}\left\lceil A_{F} \backslash \Pi_{E} .\right.\right.
$$

To prove the "if" part, assume that condition $\left(22\right.$ holds. To prove that $\mathcal{F}_{E} \subseteq \mathcal{F}_{F}$, fix any set $\Omega \in \mathcal{F}_{E}$ and using Lemma 4 find a finite set $L \subseteq \Pi \backslash A_{E}$ such that

$$
\bigcap_{p \in L} p \mathbb{Z}^{\bullet} \cap \bigcap_{p \in A_{E} \backslash \Pi_{E}}\left(\left\{0, \alpha_{E}(p)\right\}+p \mathbb{Z}\right) \subseteq \Omega .
$$

Consider the finite set

$$
\Lambda=\left(L \cup A_{E}\right) \backslash A_{F}=L \cup\left(A_{E} \backslash A_{F}\right) \supseteq L
$$

and observe that condition (2) implies the inclusion

$$
\mathcal{F}_{F} \ni \bigcap_{p \in \Lambda} p \mathbb{Z}^{\bullet} \cap \bigcap_{p \in A_{F} \backslash \Pi_{F}}\left(\left\{0, \alpha_{F}(p)\right\}+p \mathbb{Z}\right) \subseteq \bigcap_{p \in L} p \mathbb{Z}^{\bullet} \cap \bigcap_{p \in A_{E} \backslash \Pi_{E}}\left(\left\{0, \alpha_{E}(p)\right\}+p \mathbb{Z}\right) \subseteq \Omega,(3)
$$

yielding $\Omega \in \mathcal{F}_{F}$.

Lemma 6. For two nonempty subsets $E, F \subseteq \mathbb{N}$ with $\min \{|E|,|F|\}=1$ the relation $\mathcal{F}_{E} \subseteq \mathcal{F}_{F}$ holds if and only if $|E|=1$ and $E \subseteq F$.

Proof. The "if" part is trivial. To prove the "only if" part, assume that $\mathcal{F}_{E} \subseteq \mathcal{F}_{F}$. First we prove that $|E|=1$. Assuming that $|E|>1$ and taking into account that $\min \{|E|,|F|\}=1$, we conclude that $|F|=1$. Choose a prime number $p>\max (E \cup F)$. Since $\bigcap_{y \in E} \overline{y+p \mathbb{Z}} \in \mathcal{F}_{E} \subseteq \mathcal{F}_{F}$, for the unique number $x$ in the set $F$, there exists a

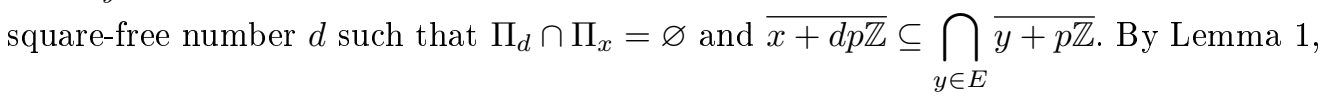

$$
x+q p \mathbb{Z}^{\bullet} \subseteq \overline{x+d p \mathbb{Z}} \subseteq \bigcap_{y \in E} \overline{y+p \mathbb{Z}}=\bigcap_{y \in E}(\{0, y\}+p \mathbb{Z})=p \mathbb{Z} .
$$

The latter equality follows from $p>\max E$ and $|E|>1$. Then $x+d p \mathbb{Z} \bullet \subseteq p \mathbb{Z}$ implies $x \in p \mathbb{Z}$, which contradicts the choice of $p>\max (E \cup F) \geq x$. This contradiction shows that $|E|=1$. Let $z$ be the unique element of the set $E$. 
It remains to prove that $z \in F$. To derive a contradiction, assume that $z \notin F$. Take any odd prime number $p>\max (E \cup F)$ and consider the set

$$
\{0, z\}+p \mathbb{Z}=\overline{z+p \mathbb{Z}} \in \mathcal{F}_{E} \subseteq \mathcal{F}_{F} .
$$

By the definition of the filter $\mathcal{F}_{F}$, for every $x \in F$ there exists a square-free number $d_{x}$ such that $\Pi_{d_{x}} \cap \Pi_{x}=\varnothing$ and

$$
\bigcap_{x \in F} \overline{x+d_{x} \mathbb{Z}} \subseteq \overline{z+p \mathbb{Z}}=\{0, z\}+p \mathbb{Z} .
$$

Consider the set $P=\bigcup_{x \in F} \Pi_{d_{x}}$. If $p \in \Pi_{d_{x}}$ for some $x \in F$, we can use the Chinese Remainder Theorem 2 and find a number

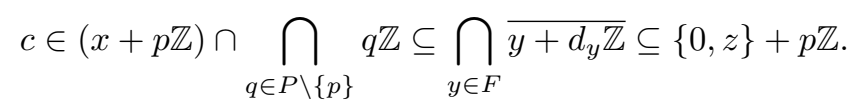

Taking into account that $x$ is not divisible by $p$, we conclude that $c \in(x+p \mathbb{Z}) \cap(z+p \mathbb{Z})$ and hence $x-z \in p \mathbb{Z}$, which contradicts the choice of $p>\max (E \cup F)$. This contradiction shows that $p \notin P$. Since $p \geq 3$, we can find a number $z^{\prime} \notin\{0, z\}+p \mathbb{Z}$ and using the Chinese Remainder Theorem 2 , find a number

$$
u \in\left(z^{\prime}+p \mathbb{Z}\right) \cap \bigcap_{q \in P} q \mathbb{Z} \bullet \subseteq \bigcap_{y \in F} \overline{y+d_{y} \mathbb{Z}} \subseteq\{0, z\}+p \mathbb{Z},
$$

which is a desired contradiction showing that $E \subseteq F$.

As we know, the largest element of the superconnecting poset $\mathfrak{F}$ is the superconnecting filter $\mathcal{F}_{\infty}$. This filter can be characterized as follows.

Lemma 7. The superconnecting filter $\mathcal{F}_{\infty}$ of the Kirch space is generated by the base consisting of the sets $q \mathbb{N}$ for an odd square-free number $q \in \mathbb{N}$, i.e.

$$
\mathcal{F}_{\infty}=\left\{B \subseteq \mathbb{Z}^{\bullet}: \exists q \in(2 \mathbb{N}-1) \backslash \bigcup_{p \in \Pi} p^{2} \mathbb{N}(q \mathbb{Z} \bullet B)\right\} .
$$

Proof. Lemma 1 implies that each element $F \in \mathcal{F}_{\infty}$ contains the set $q \mathbb{Z} \bullet$ for some odd square-free number $q$. Conversely, let $q$ be an odd square-free number. Then the sets $U_{1}=1+q \mathbb{Z}$ and $U_{2}=2+q \mathbb{Z}$ are open in the Kirch topology on $\mathbb{Z} \bullet$. By Lemma 1 we have

$$
\overline{U_{1}} \cap \overline{U_{2}}=\mathbb{Z} \bullet \bigcap_{p \in \Pi_{q}}(\{0,1\}+p \mathbb{Z}) \cap(\{0,2\}+p \mathbb{Z})=\mathbb{Z} \bullet \bigcap_{p \in \Pi_{q}} p \mathbb{Z}=q \mathbb{Z} \bullet .
$$

Hence $q \mathbb{Z} \bullet \in \mathcal{F}_{\infty}$.

Lemma 8. For a nonempty subset $E \subseteq \mathbb{Z} \bullet$ the following conditions are equivalent:

(1) $\mathcal{F}_{E}=\mathcal{F}_{\infty}$;

(2) $A_{E}=\{2\}$.

If $|E|=2$, then conditions (1), (2) are equivalent to

(3) $E \in\left\{\left\{2^{n}, 2^{n+1}\right\},\left\{-2^{n},-2^{n+1}\right\},\left\{-2^{n}, 2^{n}\right\}: n \in \omega\right\}$. 
Proof. (1) $\Rightarrow(2)$ : Assume $\mathcal{F}_{E}=\mathcal{F}_{\infty}$. Consider the set $F=\{1,2\}$ and observe that $A_{F}=\Pi_{1} \cup \Pi_{2} \cup \Pi_{2-1}=\{2\}$ and $\Pi_{F}=\varnothing$. Thus $A_{F} \subseteq A_{E}, \Pi_{F} \backslash\{2\} \subseteq \Pi_{E}$ and $\alpha_{F}\left\lceil A_{F} \backslash \Pi_{E}=\alpha_{E}\left\lceil A_{F} \backslash \Pi_{E}\right.\right.$. Lemma 5 implies $\mathcal{F}_{E} \subseteq \mathcal{F}_{F}$. Since $\mathcal{F}_{E}=\mathcal{F}_{\infty}$ is the largest element of $\mathfrak{F}$ we get $\mathcal{F}_{E}=\mathcal{F}_{F}$. By using again Lemma 5 we get $A_{E} \subseteq A_{F}$ which implies that $A_{E}=\{2\}$.

(2) $\Rightarrow(1)$ : If $A_{E}=\{2\}$, then by the Lemma 4 the filter $\mathcal{F}_{E}$ is generated by the base consisting of the sets $q \mathbb{Z} \bullet$ for an odd square-free number $q \in \mathbb{Z} \bullet$. Therefore $\mathcal{F}_{E}=\mathcal{F}_{\infty}$ by the Lemma 7 .

$(2) \Rightarrow(3)$ : Assume that $|E|=2$ and $A_{E}=\{2\}$. By Lemma $2 E=\left\{\varepsilon 2^{a}, \delta 2^{b}\right\}$, where $a, b \in \omega$ and $\varepsilon, \delta \in\{-1,1\}$. Without loss of generality we can assume that $b \leqslant a$. By Lemma 2, $\Pi_{\varepsilon 2^{a}-\delta 2^{b}}=\Pi_{\varepsilon 2^{b}\left(2^{a-b}-\delta / \varepsilon\right)} \subseteq\{2\}$. The last inclusion implies that $a-b=1$ and $\delta / \varepsilon=1$ or $a-b=0$ and $\delta / \varepsilon=-1$. In the first case the set $E$ equals $\left\{2^{b}, 2^{b+1}\right\}$ or $\left\{-2^{b},-2^{b+1}\right\}$, in the second case $E=\left\{2^{b},-2^{b}\right\}$.

$(3) \Rightarrow(2)$ : The implication (3) $\Rightarrow(2)$ follows from Lemma 2

In the following lemmas by $\mathfrak{F}^{\prime}$ we denote the set of maximal elements of the poset $\mathfrak{F} \backslash\left\{\mathcal{F}_{\infty}\right\}$.

Lemma 9. For a nonempty finite subset $E \subseteq \mathbb{Z} \bullet$ the filter $\mathcal{F}_{E}$ belongs to the family $\mathfrak{F}^{\prime}$ if and only if there exists an odd prime number $p \notin \Pi_{E}$ such that $A_{E}=\{2, p\}$.

Proof. To prove the "if" part, assume that $A_{E}=\{2, p\}$ and $p \notin \Pi_{E}$ for some odd prime number $p$. By Lemma $8, \mathcal{F}_{E} \neq \mathcal{F}_{\infty}$. To show that the filter $\mathcal{F}_{E}$ is maximal in $\mathfrak{F} \backslash\left\{\mathcal{F}_{\infty}\right\}$, take any finite set $F \subset \mathbb{Z} \bullet$ such that $\mathcal{F}_{E} \subseteq \mathcal{F}_{F} \neq \mathcal{F}_{\infty}$. By Lemmas 5 and 8 , $\{2\} \neq A_{F} \subseteq\{2, p\}, \Pi_{F} \subseteq \Pi_{E} \cup\{2\}=\{2\}$, and $\alpha_{F}\left\lceil A_{F} \backslash \Pi_{E}=\alpha_{E}\left\lceil A_{F} \backslash \Pi_{E}\right.\right.$. It follows that $A_{F}=\{2, p\}=A_{E}, \Pi_{F} \cup\{2\}=\Pi_{E} \cup\{2\}$ and $\alpha_{F}=\alpha_{E}$. Applying Lemma 5 we conclude that $\mathcal{F}_{E}=\mathcal{F}_{F}$, which means that the filter $\mathcal{F}_{E}$ is a maximal element of the poset $\mathcal{F} \backslash\left\{\mathcal{F}_{\infty}\right\}$.

To prove the "only if" part, assume that $\mathcal{F}_{E} \in \mathfrak{F}^{\prime}$. By Lemma 8, $A_{E} \neq\{2\}$ and hence there exists an odd prime number $p \in A_{E}$. We claim that $p \notin \Pi_{E}$. To derive a contradiction, assume that $p \in \Pi_{E}$ and consider the sets $F=\{p, 2 p\}$ and $G=\{1, p, 2 p\}$. By Lemma 2, $A_{F}=A_{G}=\{2, p\}, \Pi_{F}=\{p\}$, and $\Pi_{G}=\varnothing$. Taking into account that $F \subset G, A_{F}=\{2, p\} \subseteq A_{E}, \Pi_{F} \backslash\{2\}=\{p\} \subseteq \Pi_{E}$ and $A_{F} \backslash \Pi_{E} \subseteq\{2\}$, we can apply Lemmas 5,8 and conclude that $\mathcal{F}_{E} \subseteq \mathcal{F}_{F} \subseteq \mathcal{F}_{G} \neq \mathcal{F}_{\infty}$. The maximality of $\mathcal{F}_{E}$ implies $\mathcal{F}_{E}=\mathcal{F}_{F}=\mathcal{F}_{G}$. By Lemma 5 the equality $\mathcal{F}_{G}=\mathcal{F}_{F}$ implies $p \in \Pi_{F} \backslash\{2\} \subseteq \Pi_{G}=\varnothing$, which is a contradiction showing that $p \notin \Pi_{E}$.

Now consider the set $H=\left\{\alpha_{E}(p), p, 2 p\right\}$ and observe that $A_{H}=\{2, p\}, \Pi_{H}=\varnothing$ and $\alpha_{H}(p)=\alpha_{E}(p)$. Lemmas 5 and 8 guarantee that $\mathcal{F}_{E} \subseteq \mathcal{F}_{H} \neq \mathcal{F}_{\infty}$. By the maximality of $\mathcal{F}_{E}$, we have $\mathcal{F}_{E}=\mathcal{F}_{H}$. Applying Lemma 5 once more, we conclude that $A_{E}=A_{H}=$ $\{2, p\}$.

Lemma 9 implies the following description of the set $\mathfrak{F}^{\prime}$.

Lemma 10. $\mathfrak{F}^{\prime}=\left\{\mathcal{F}_{\{a, p, 2 p\}}: p \in \Pi \backslash\{2\}, a \in\{1, \ldots, p-1\}\right\}$.

Let $\mathfrak{F}^{\prime \prime}$ be the set of maximal elements of the poset $\mathfrak{F} \backslash\left(\mathfrak{F}^{\prime} \cup\left\{\mathcal{F}_{\infty}\right\}\right)$

Lemma 11. For a finite set $E \subset \mathbb{Z} \bullet$, the filter $\mathcal{F}_{E}$ belongs to the family $\mathfrak{F}^{\prime \prime}$ if and only if one of the following conditions holds: 
(1) there exists an odd prime number $p$ such that $p \in \Pi_{E}$ and $A_{E}=\{2, p\}$;

(2) there are two distinct odd prime numbers $p, q$ such that $A_{E}=\{2, p, q\}$ and $\Pi_{E} \subseteq$ $\{2\}$.

Proof. To prove the "only if" part, assume that $\mathcal{F}_{E} \in \mathfrak{F}^{\prime \prime}$. By Lemma 8 there is an odd prime number $p \in A_{E}$. If $A_{E}=\{2, p\}$, then $p \in \Pi_{E}$ by Lemma 9, and condition (1) is satisfied. So, we assume that $\{2, p\} \neq A_{E}$ and find an odd prime number $q \in A_{E} \backslash\{2, p\}$. By Lemma 3 there is a number $x \in \mathbb{N}$ such that for the set $F=\{x, p q, 2 p q\}$ we have $A_{F}=\{2, p, q\}, \Pi_{F}=\varnothing, \alpha_{F}(p)=\alpha_{E}(p)$ and $\alpha_{F}(q)=\alpha_{E}(q)$. Then $\mathcal{F}_{E} \subseteq \mathcal{F}_{F}$ by Lemma 5 and $\mathcal{F}_{F} \in \mathfrak{F} \backslash\left(\mathfrak{F}^{\prime} \cup\left\{\mathcal{F}_{\infty}\right\}\right)$ by Lemma 9. Now the maximality of the filter $\mathcal{F}_{E}$ implies that $\mathcal{F}_{E}=\mathcal{F}_{F}$ and hence $A_{E}=A_{F}=\{2, p, q\}$ and $\Pi_{E} \subseteq \Pi_{F} \cup\{2\}=\{2\}$, see Lemma 5 .

To prove the "if" part, we consider two cases. First we assume that $A_{E}=\{2, p\}$ and $p \in \Pi_{E}$ for some odd prime number $p$. By Lemmas 8 and $9, \mathcal{F}_{E} \in \mathfrak{F} \backslash\left(\left\{\mathcal{F}_{\infty}\right\} \cup \mathfrak{F}^{\prime}\right)$. To prove that $\mathcal{F}_{E}$ is a maximal element of $\mathfrak{F} \backslash\left(\left\{\mathcal{F}_{\infty}\right\} \cup \mathfrak{F}^{\prime}\right)$, take any finite set $F \subseteq \mathbb{Z}$ such that $\mathcal{F}_{E} \subseteq \mathcal{F}_{F} \in \mathfrak{F} \backslash\left(\left\{\mathcal{F}_{\infty}\right\} \cup \mathfrak{F}^{\prime}\right)$. Lemma 6 implies that $\min \{|E|,|F|\} \geq 2$ and then by Lemmas 5 and 9 , we have $A_{F}=\{2, p\}, \Pi_{F} \backslash\{2\} \subseteq\{p\}$ and $\alpha_{E}\left\lceil A_{F} \backslash\{p\}=\alpha_{F}\left\lceil A_{F} \backslash\{p\}\right.\right.$. Now notice that $p \in \Pi_{F}$ since otherwise $\mathcal{F}_{F} \in \mathfrak{F}^{\prime}$ by Lemma 9 . By using again Lemma 5 we get $\mathcal{F}_{F}=\mathcal{F}_{E}$ which means that $\mathcal{F}_{E} \in \mathfrak{F}^{\prime \prime}$.

Now assume that there are two distinct odd prime numbers $p, q$ such that $A_{E}=$ $\{2, p, q\}$ and $\Pi_{E} \subseteq\{2\}$. By Lemmas 8 and $9, \mathcal{F}_{E} \in \mathfrak{F} \backslash\left(\left\{\mathcal{F}_{\infty}\right\} \cup \mathfrak{F}^{\prime}\right)$. To prove that $\mathcal{F}_{E}$ is a maximal element of $\mathfrak{F} \backslash\left(\left\{\mathcal{F}_{\infty}\right\} \cup \mathfrak{F}^{\prime}\right)$, take any finite set $F \subseteq \mathbb{Z} \bullet$ such that $\mathcal{F}_{E} \subseteq \mathcal{F}_{F} \in \mathfrak{F} \backslash\left(\left\{\mathcal{F}_{\infty}\right\} \cup \mathfrak{F}^{\prime}\right)$. Lemma 5 implies that $A_{F} \subseteq\{2, p, q\}, \Pi_{F} \subseteq\{2\}$ and $\alpha_{E}\left\lceil A_{F} \backslash \Pi_{E}=\alpha_{F}\left\lceil A_{F} \backslash \Pi_{E}\right.\right.$. Taking into account that $\mathcal{F}_{F} \notin \mathfrak{F}^{\prime} \cup\left\{\mathcal{F}_{\infty}\right\}$ and $\Pi_{F} \subseteq\{2\}$, we can apply Lemmas 9,8 and conclude that $A_{F}=\{2, p, q\}$. We therefore know that $A_{F}=A_{E}, \Pi_{E} \cup\{2\}=\Pi_{F} \cup\{2\}$ and $\alpha_{F} \mid A_{E} \backslash \Pi_{F}=\alpha_{E}\left\lceil A_{E} \backslash \Pi_{F}\right.$. By Lemma $5 \mathcal{F}_{E}=\mathcal{F}_{F}$ and hence $\mathcal{F}_{E} \in \mathfrak{F}^{\prime \prime}$.

Lemma 12. For any homeomorphism $h$ of the Kirch space and any odd prime number $p$ we have

$$
\tilde{h}\left(\mathcal{F}_{\{p, 2 p\}}\right)=\mathcal{F}_{\{p, 2 p\}} .
$$

Proof. By Proposition 1 the homeomorphism $h$ induces an order isomorphism $\tilde{h}$ of the superconnecting poset $\mathfrak{F}$ on the Kirch space. Then $\tilde{h}\left[\mathfrak{F}^{\prime}\right]=\mathfrak{F}^{\prime}$ and $\tilde{h}\left[\mathfrak{F}^{\prime \prime}\right]=\mathfrak{F}^{\prime \prime}$.

By Lemmas 11 and $3, \mathfrak{F}^{\prime \prime}=\mathfrak{F}_{2}^{\prime \prime} \cup \mathfrak{F}_{3}^{\prime \prime}$ where

$$
\begin{aligned}
& \mathfrak{F}_{2}^{\prime \prime}=\left\{\mathcal{F}_{\{p, 2 p\}}: p \in \Pi \backslash\{2\}\right\} \quad \text { and } \\
& \mathfrak{F}_{3}^{\prime \prime}=\left\{\mathcal{F}_{\{x, p q, 2 p q\}}: p, q \in \Pi \backslash\{3\}, x \in\{0, \ldots, p q-1\} \backslash(p \mathbb{Z} \cup q \mathbb{Z})\right\} .
\end{aligned}
$$

By Lemmas 5 and 9 for every filter $\mathcal{F}_{\{p, 2 p\}} \in \mathfrak{F}_{2}^{\prime \prime}$ the set

$$
\uparrow \mathcal{F}_{\{p, 2 p\}}=\left\{\mathcal{F} \in \mathfrak{F}^{\prime}: \mathcal{F}_{\{p, 2 p\}} \subset \mathcal{F}_{E}\right\}
$$

coincides with the set $\left\{\mathcal{F}_{\{a, p, 2 p\}}: a \in\{1, \ldots, p-1\}\right\}$ and hence has cardinality $p-1$.

On the other hand, for any filter $\mathcal{F}_{\{x, p q, 2 p q\}} \in \mathfrak{F}_{3}^{\prime \prime}$, the set

$$
\uparrow \mathcal{F}_{\{x, p q, 2 p q\}}=\left\{\mathcal{F} \in \mathfrak{F}^{\prime}: \mathcal{F}_{\{x, p q, 2 p q\}} \subset \mathcal{F}\right\}
$$

coincides with the doubleton $\left\{\mathcal{F}_{\{x, p, 2 p\}}, \mathcal{F}_{\{x, q, 2 q\}}\right\}$. 
These order properties uniquely determine the filters $\mathcal{F}_{\{p, 2 p\}}$ for $p \in \Pi \backslash\{3\}$ and ensure that $\tilde{h}\left(\mathcal{F}_{\{p, 2 p\}}\right)=\mathcal{F}_{\{p, 2 p\}}$ for every $p \in \Pi \backslash\{3\}$.

Next, observe that $\mathcal{F}_{\{3,6\}}$ is a unique element $\mathcal{F}$ of $\mathfrak{F}^{\prime \prime}$ such that

$$
\uparrow \mathcal{F} \cap \bigcup_{p \in \Pi \backslash\{3\}} \uparrow \mathcal{F}_{\{p, 2 p\}}=\varnothing .
$$

This uniqueness order property of $\mathcal{F}_{\{3,6\}}$ ensures that $\tilde{h}\left(\mathcal{F}_{\{3,6\}}\right)=\mathcal{F}_{\{3,6\}}$.

Lemma 13. Let $E \subseteq \mathbb{Z} \bullet$ be a finite subset such that $A_{E}=\{2, p\}$ for some odd prime number $p \notin \Pi_{E}$. Then $A_{h[E]}=\{2, p\}$.

Proof. By Lemma $9 \mathcal{F}_{E} \in \mathfrak{F}^{\prime}$. Consider the doubleton $\{p, 2 p\}$ which has $A_{\{p, 2 p\}}=\{2, p\}$ and $\Pi_{\{p, 2 p\}}=\{p\}$. By Lemma $5, \mathcal{F}_{\{p, 2 p\}} \subseteq \mathcal{F}_{E}$ and by Lemma 12 .

$$
\mathcal{F}_{\{p, 2 p\}}=\tilde{h}\left(\mathcal{F}_{\{p, 2 p\}}\right)=\mathcal{F}_{\{h(p), h(2 p)\}} \subseteq \mathcal{F}_{h[E]} .
$$

By Lemma 5, $A_{h[E]} \subseteq A_{\{p, 2 p\}}=\{2, p\}$. By Lemma $8, A_{h[E]}=\{2, p\}$.

Definition 1. A homeomorphism $h$ of the Kirch space $\left(\mathbb{Z}^{\bullet}, \tau_{K}\right)$ is called positive if $h(1)>0$.

Lemma 14. Every positive homeomorphism $h$ of the Kirch space has $h(x)=x$ for any $x \in\left\{ \pm 2^{n}, n \in \omega\right\}$

Proof. Consider the graph $\Gamma_{2}=\left(V_{2}, \mathcal{E}\right)$ with the set of vertices $V_{2}=\left\{ \pm 2^{n}: n \in \omega\right\}$ and the set of edges

$$
\mathcal{E}=\left\{\left\{2^{n}, 2^{n+1}\right\},\left\{-2^{n},-2^{n+1}\right\},\left\{-2^{n}, 2^{n}\right\}: n \in \omega\right\} .
$$

Observe that 1 and -1 are the unique vertices of $\Gamma_{2}$ that have order 2 . Any other vertices have order 3 . This ensures that $h(1)= \pm 1$. The positivity of $h$ implies that $h(1)=1$. Then $h(-1)=-1, h(2)=2$. Hence $h\left( \pm 2^{n}\right)= \pm 2^{n}$ for all $n \in \omega$.

Lemmas 14 and 12 imply

Lemma 15. For any positive homeomorphism $h$ of the Kirch space and any odd prime number $p$ we have

$$
\tilde{h}\left(\mathcal{F}_{\{1, p, 2 p\}}\right)=\mathcal{F}_{\{1, p, 2 p\}} \quad \text { and } \quad \tilde{h}_{\left(\mathcal{F}_{\{2, p, 2 p\}}\right)=\mathcal{F}_{\{2, p, 2 p\}} .}
$$

Lemma 16. For an integer number $x \in \mathbb{Z} \bullet\{-2,-1,1,2\}$ and an odd prime $p$, the following conditions are equivalent:

(1) $p \in \Pi_{x}$;

(2) $\mathcal{F}_{\{1, x\}} \subseteq \mathcal{F}_{\{1, p, 2 p\}}$ and $\mathcal{F}_{\{2, x\}} \subseteq \mathcal{F}_{\{2, p, 2 p\}}$.

Proof. If $p \in \Pi_{x}$, then $A_{\{1, p, 2 p\}}=\{2, p\} \subseteq A_{\{1, x\}}, \Pi_{\{1, x\}} \cup\{2\}=\{2\}=\Pi_{\{1, p, 2 p\}} \cup\{2\}$ and $\alpha_{\{1, x\}}(p)=1=\alpha_{\{1, p, 2 p\}}(p)$. By Lemma 5 . $\mathcal{F}_{\{1, x\}} \subseteq \mathcal{F}_{\{1, p, 2 p\}}$. By analogy we can prove that $\mathcal{F}_{\{2, x\}} \subseteq \mathcal{F}_{\{2, p, 2 p\}}$. we have

Conversely, assume $\mathcal{F}_{\{1, x\}} \subseteq \mathcal{F}_{\{1, p, 2 p\}}$ and $\mathcal{F}_{\{2, x\}} \subseteq \mathcal{F}_{\{2, p, 2 p\}}$. By Lemmas 5 and 2 $\{2, p\}=A_{\{1, p, 2 p\}} \subseteq A_{\{1, x\}}=\Pi_{x} \cup \Pi_{x-1}$ and $\{2, p\}=A_{\{2, p, 2 p\}} \subseteq A_{\{2, x\}}=\{2\} \cup \Pi_{x} \cup \Pi_{x-2}$ and hence $p \in\left(\Pi_{x} \cup \Pi_{x-1}\right) \cap\left(\Pi_{x} \cup \Pi_{x-2}\right) \backslash\{2\} \subseteq \Pi_{x}$. 
Proposition 1 and Lemmas $14,15,16$ imply

Lemma 17. For every homeomorphism $h$ of the Kirch space and any number $x \in \mathbb{N}$ we have

$$
\Pi_{x} \cup\{2\}=\Pi_{h(x)} \cup\{2\} .
$$

For every prime number $p$ consider the set

$$
V_{p}=\left\{ \pm 2^{n-1} p^{m}: n, m \in \mathbb{N}\right\}
$$

of numbers $x \in \mathbb{N}$ such that $p \in \Pi_{x} \subseteq\{2, p\}$. Lemmas 14 and 17 imply that $h\left[V_{p}\right]=V_{p}$ for every homeomorphism $h$ of the Kirch space.

Consider the graph $\Gamma_{p}=\left(V_{p}, \mathcal{E}_{p}\right)$ on the set $V_{p}$ with the set of edges

$$
\mathcal{E}_{p}:=\left\{E \in\left[V_{p}\right]^{2}: A_{E}=\{2, p\}\right\} .
$$

Lemma 18. For every prime number $p$ and every homeomorphism $h$ of the Kirch space, the restriction of $h$ to $V_{p}$ is an isomorphism of the graph $\Gamma_{p}$.

Proof. Let $E \in \mathcal{E}_{p}$. Since $p \in \Pi_{E}$, we can apply Lemma 11 and conclude that $\mathcal{F}_{E} \in \mathfrak{F}^{\prime \prime}$. Using fact that $\tilde{h}$ is isomorphism of $\mathfrak{F}$ we get $\mathcal{F}_{h[E]}=\tilde{h}\left(\overline{\mathcal{F}}_{E}\right) \in \mathfrak{F}^{\prime \prime}$. Since $h[E] \subseteq h\left[V_{p}\right]=$ $V_{p}$, we obtain $p \in \Pi_{h[E]}$. Using Lemma 11 once more, we obtain that $A_{h[E]}=\{2, p\}$, which means that $h[E] \in \mathcal{E}_{p}$. By analogical reasoning we can prove that $h^{-1}[E] \in \mathcal{E}_{p}$ for every $E \in \mathcal{E}_{p}$. This means that $h \uparrow V_{p}$ is an isomorphism of the graph $\Gamma_{p}$.

The structure of the graph $\Gamma_{p}$ depends on properties of the prime number $p$.

A prime number $p$ is called

- Fermat prime if $p=2^{n}+1$ for some $n \in \mathbb{N}$;

- Mersenne prime if $p=2^{n}-1$ for some $n \in \mathbb{N}$;

- Fermat-Mersenne if $p$ is either Fermat prime or Mersenne prime.

It is known (and easy to see) that for any Fermat prime number $p=2^{n}+1$ the exponent $n$ is a power of 2 , and for any Mersenne prime number $p=2^{n}-1$ the power $n$ is a prime number. It is not known whether there are infinitely many Fermat-Mersenne prime numbers. All known Fermat prime numbers are the numbers $2^{2^{n}}+1$ for $0 \leq n \leq 4$ (see oeis.org/A019434 in [9]). At the moment only 51 Mersenne prime numbers are known, see the sequence oeis.org/A000043 in [9].

Lemma 19. Let $p$ be an odd prime number, $p \neq 3$.

(1) If $p=3$, then the set $\mathcal{E}_{p}$ of the edges of the graph $\Gamma_{p}$ coincides with the set of doubletons $\left\{\varepsilon 2^{a-1} 3^{b}, \varepsilon 2^{a-1} 3^{b+1}\right\},\left\{\varepsilon 2^{a-1} 3^{b}, 2 \varepsilon^{a-1} 3^{b+2}\right\},\left\{\varepsilon 2^{a-1} 3^{b}, \varepsilon 2^{a} 3^{b}\right\}$, $\left\{\varepsilon 2^{a-1} 3^{b}, \varepsilon 2^{a+1} 3^{b}\right\},\left\{\varepsilon 2^{a-1} 3^{b+1}, \varepsilon 2^{a+1} 3^{b}\right\},\left\{\varepsilon 2^{a+1} 3^{b}, \varepsilon 2^{a} 3^{b+1}\right\},\left\{\varepsilon 2^{a+3} 3^{b}, \varepsilon 2^{a} 3^{b+2}\right\}$, $\left\{\varepsilon 2^{a-1} 3^{b},-\varepsilon 2^{a-1} 3^{b+1}\right\},\left\{\varepsilon 2^{a-1} 3^{b},-\varepsilon 2^{a} 3^{b}\right\},\left\{\varepsilon 2^{a-1} 3^{b},-\varepsilon 2^{a+2} 3^{b}\right\}$, $\left\{\varepsilon 2^{a-1} 3^{b},-\varepsilon 2^{a-1} 3^{b}\right\}$ for some $a, b \in \mathbb{N}, \varepsilon \in\{-1,1\}$.

(2) If $p=2^{m}+1>3$ is Fermat prime, then

$$
\begin{aligned}
\mathcal{E}_{p}=\{ & \left\{\varepsilon 2^{a-1} p^{b}, \varepsilon 2^{a-1} p^{b+1}\right\},\left\{\varepsilon 2^{a-1} p^{b}, \varepsilon 2^{a} p^{b}\right\},\left\{\varepsilon 2^{a-1} p^{b},-\varepsilon 2^{a+m-1} p^{b}\right\}, \\
& \left.\left\{\varepsilon 2^{a-1} p^{b},-\varepsilon 2^{a-1} p^{b}\right\},\left\{\varepsilon 2^{m+a-1} p^{b}, \varepsilon 2^{a-1} p^{b+1}\right\}: a, b \in \mathbb{N}, \varepsilon \in\{-1,1\}\right\} .
\end{aligned}
$$


(3) If $p=2^{m}-1>3$ is a Mersenne prime, then

$$
\begin{aligned}
\mathcal{E}_{p}=\{ & \left\{\varepsilon 2^{a} p^{b}, \varepsilon 2^{a-1} p^{b}\right\},\left\{\varepsilon 2^{a-1} p^{b}, \varepsilon 2^{m+a-1} p^{b}\right\},\left\{\varepsilon 2^{a-1} p^{b+1}, \varepsilon 2^{m+a-1} p^{b}\right\}, \\
& \left.\left\{\varepsilon 2^{a-1} p^{b},-\varepsilon 2^{a-1} p^{b}\right\},\left\{\varepsilon 2^{a-1} p^{b},-\varepsilon 2^{a-1} p^{b+1}\right\}: a, b \in \mathbb{N}, \varepsilon \in\{-1,1\}\right\} .
\end{aligned}
$$

(4) If $p$ is not Fermat-Mersenne, then

$$
\mathcal{E}_{p}=\left\{\left\{\varepsilon 2^{a-1} p^{b},-\varepsilon 2^{a-1} p^{b}\right\},\left\{\varepsilon 2^{a-1} p^{b}, \varepsilon 2^{a} p^{b}\right\}: a, b \in \mathbb{N}, \varepsilon \in\{-1,1\}\right\} .
$$

Proof. Proof of Lemma 19 in each of cases (1)-(4) will be similar. The edges of graph $\Gamma_{p}$ are 2-element subsets of set $V_{p}$ such that $A_{E}=\{2, p\}$. Since the vertices of graph $\Gamma_{p}$ are numbers of the form $\pm 2^{n-1} p^{m}$, where $n, m \in \mathbb{N}$, we can apply Lemma 2 and conclude that a doubleton $\{x, y\} \subset V_{p}$ belongs to $\mathcal{E}_{p}$ if and only if $\{2, p\}=\Pi_{x} \cup \Pi_{y} \cup \Pi_{x-y}$. In subsequent proofs, we will intensively use the Mihăilescu Theorem 4 saying that $2^{3}, 3^{2}$ is a unique pair of consecutive powers.

1. First we consider the case of $p=3$. It is easy to see that the doubletons $\{x, y\}$ written in statement (1) have $\Pi_{x} \cup \Pi_{y} \cup \Pi_{x-y} \subseteq\{2,3\}$, which implies that $\{x, y\} \in \mathcal{E}_{3}$. It remains to show that every doubleton $\{x, y\} \in \mathcal{E}_{3}$ is of the form indicated in statement (1). Write $\{x, y\}$ as $\left\{\varepsilon 2^{a-1} 3^{b}, \delta 2^{c-1} 3^{d}\right\}$ for some $a, b, c, d \in \mathbb{N}, \varepsilon, \delta \in\{-1,1\}$ such that $2^{a-1} 3^{b} \leq 2^{c-1} 3^{d}$.

If $a=c$ and $b=d$, then $\varepsilon \neq \delta$ and $\{x, y\}=\left\{\varepsilon 2^{a-1} 3^{b},-\varepsilon 2^{a-1} 3^{b}\right\}$.

If $a=c$, then $b \leq d$ and the inclusion $\Pi_{x-y} \subseteq\{2,3\}$ implies that $\Pi_{3^{d-b}-\varepsilon / \delta} \subseteq\{2,3\}$ and hence $3^{d-b}-\varepsilon / \delta$ is a power of 2 . If $\varepsilon / \delta=1$ then by the Mihăilescu Theorem $4, d-b \in$ $\{1,2\}$, which means that $\{x, y\}$ is equal to $\left\{\varepsilon 2^{a-1} 3^{b}, \varepsilon 2^{a-1} 3^{b+1}\right\}$ or $\left\{\varepsilon 2^{a-1} 3^{b}, \varepsilon 2^{a-1} 3^{b+2}\right\}$. If $\varepsilon / \delta=-1$ then by the Mihăilescu Theorem $4, d-b \in\{0,1\}$, which means that $\{x, y\}$ is equal to $\left\{\varepsilon 2^{a-1} 3^{b},-\varepsilon 2^{a-1} 3^{b}\right\}$ or $\left\{\varepsilon 2^{a-1} 3^{b},-\varepsilon 2^{a-1} 3^{b+1}\right\}$.

If $b=d$, then $a \leq c$ and the inclusion $\Pi_{x-y} \subseteq\{2,3\}$ implies that $\Pi_{2^{c-a}-\varepsilon / \delta} \subseteq\{2,3\}$ and hence $2^{c-a}-\varepsilon / \delta$ is either 2 or a power of 3 . If $\varepsilon / \delta=1$ then by the Mihăilescu Theorem $4 . c-a \in\{1,2\}$, which means that $\{x, y\}$ is equal to $\left\{\varepsilon 2^{a-1} 3^{b}, \varepsilon 2^{a} 3^{b}\right\}$ or $\left\{\varepsilon 2^{a-1} 3^{b}, \varepsilon 2^{a+1} 3^{b}\right\}$. If $\varepsilon / \delta=-1$ then by the Mihăilescu Theorem $4, c-a \in$ $\{0,1,3\}$, which means that $\{x, y\}$ is equal to $\left\{\varepsilon 2^{a-1} 3^{b},-\varepsilon 2^{a-1} 3^{b}\right\},\left\{\varepsilon 2^{a-1} 3^{b},-\varepsilon 2^{a} 3^{b}\right\}$ or $\left\{\varepsilon 2^{a-1} 3^{b},-\varepsilon 2^{a+2} 3^{b}\right\}$.

So, we assume that $a \neq c$ and $b \neq d$. In this case we should consider four subcases.

If $a<c$ and $b<d$, then $\Pi_{x-y} \subseteq\{2,3\}$ implies that each prime divisor of $2^{c-a} 3^{d-b}-$ $\varepsilon / \delta$ is equal to 2 or 3 , which is not possible.

If $a<c$ and $b>d$, then $\Pi_{x-y} \subseteq\{2,3\}$ and $2^{a-1} 3^{b} \leq 2^{c-1} 3^{d}$ imply that $2^{c-a}-$ $(\varepsilon / \delta) 3^{b-d}=1$ which implies that $\varepsilon=\delta$. Hence $c-a=2$ and $b-d=1$ by the Mihăilescu Theorem 4 In this case $\{x, y\}=\left\{\varepsilon 2^{a-1} 3^{d+1}, \varepsilon 2^{a+1} 3^{d}\right\}$.

If $a>c$ and $b<d$, then $\Pi_{x-y} \subseteq\{2,3\}$ and $2^{a-1} 3^{b} \leq 2^{c-1} 3^{d}$ imply that $3^{d-b}-$ $(\varepsilon / \delta) 2^{a-c}=1$. This implies that $\varepsilon / \delta=1$ and hence $\langle d-b, a-c\rangle \in\{\langle 1,1\rangle,\langle 2,3\rangle\}$ by the Mihăilescu Theorem 4 In this case $\{x, y\}$ is equal to $\left\{2^{c+1} 3^{b}, 2^{c} 3^{b+1}\right\}$ or $\left\{2^{c+3} 3^{b}, 2^{c} 3^{b+2}\right\}$.

The subcase $a>c$ and $b>d$ is forbidden by the inequality $2^{a-1} 3^{b} \leq 2^{c-1} 3^{d}$. 


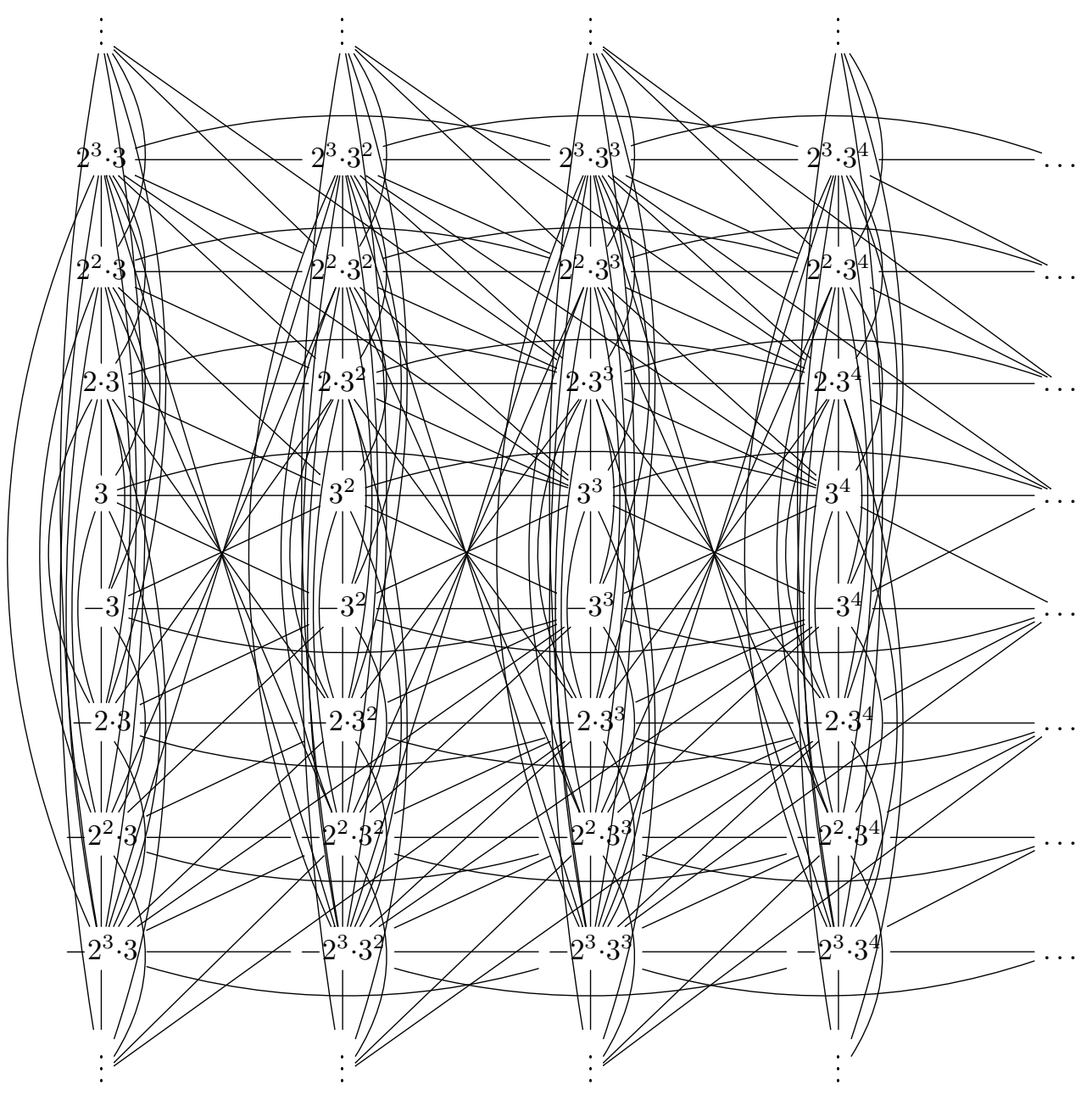

Figure 1. The graph $\Gamma_{3}$

2. Assume that $p=2^{m}+1>3$ is a Fermat prime. In this case $m>1$. Since $p>3$, $p$ is not Mersenne prime. It is easy to check that every doubleton

$$
\begin{aligned}
&\{x, y\} \in\left\{\left\{\varepsilon 2^{a-1} p^{b}, \varepsilon 2^{a-1} p^{b+1}\right\},\left\{\varepsilon 2^{a-1} p^{b}, \varepsilon 2^{a} p^{b}\right\},\left\{\varepsilon 2^{a-1} p^{b},-\varepsilon 2^{a+m-1} p^{b}\right\},\right. \\
&\left.\left\{\varepsilon 2^{a-1} p^{b},-\varepsilon 2^{a-1} p^{b}\right\},\left\{\varepsilon 2^{m+a-1} p^{b}, \varepsilon 2^{a-1} p^{b+1}\right\}: a, b \in \mathbb{N}, \varepsilon \in\{-1,1\}\right\}
\end{aligned}
$$

has $A_{\{x, y\}}=\Pi_{x} \cup \Pi_{y} \cup \Pi_{x-y}=\{2, p\}$ and hence $\{x, y\} \in \mathcal{E}_{p}$.

Now assume that $\{x, y\} \in \mathcal{E}_{p}$ is an edge of the graph $\Gamma_{p}$. Then

$$
\Pi_{x} \cup \Pi_{y} \cup \Pi_{x-y}=A_{\{x, y\}}=\{2, p\}
$$


and $\{x, y\}$ can be written as $\left\{\varepsilon 2^{a-1} p^{b}, \delta 2^{c-1} p^{d}\right\}$ for some $a, b, c, d \in \mathbb{N}, \varepsilon, \delta \in\{-1,1\}$ with $2^{a-1} p^{b} \leq 2^{c-1} p^{d}$.

If $a=c, b=d$ and $\varepsilon=-\delta$ then $\prod_{\varepsilon 2^{a-1} p^{b}-\delta 2^{a-1} p^{b}}=\prod_{\varepsilon 2^{a} p^{b}} \subset\{2, p\}$. In this case $\{x, y\}=\left\{\varepsilon 2^{a-1} p^{b},-\varepsilon 2^{a-1} p^{b}\right\}$.

If $a=c$, then $b \leq d$ and the inclusion $\Pi_{x-y} \subseteq\{2, p\}$ implies that $\Pi_{p^{d-b}-\varepsilon / \delta} \subseteq\{2, p\}$ and hence $p^{d-b}-\varepsilon / \delta$ is a power of 2 . By the Mihăilescu Theorem $4, d-b \in\{0,1\}$. If $d-b=0$, then $\varepsilon=-\delta$ and $\{x, y\}=\left\{\varepsilon 2^{a-1} p^{b},-\varepsilon 2^{a-1} p^{b}\right\}$ by the preceding case. So, we assume that $d-b=1$. Since $p$ is not Mersenne prime, we conclude that $\varepsilon=\delta$, and hence $\{x, y\}$ is equal to $\left\{\varepsilon 2^{a-1} p^{b}, \varepsilon 2^{a-1} p^{b+1}\right\}$.

If $b=d$, then $a \leq c$ and the inclusion $\Pi_{x-y} \subseteq\{2, p\}$ implies that $\Pi_{2^{c-a}-\varepsilon / \delta} \subseteq\{2, p\}$ and hence $2^{c-a}-\varepsilon / \delta$ is a power of $p$. By the Mihăilescu Theorem $4,2^{c-a}-\varepsilon / \delta \in\{1, p\}=$ $\left\{1,2^{m}+1\right\}$. If $\varepsilon=\delta$ then $c-a=1$, which means that $\{x, y\}$ is equal to $\left\{\varepsilon 2^{a-1} p^{b}, \varepsilon 2^{a} p^{b}\right\}$. If $\varepsilon=-\delta$ then $c-a=m$ and $\{x, y\}=\left\{\varepsilon 2^{a-1} p^{b},-\varepsilon 2^{a+m-1} p^{b}\right\}$.

So, we assume that $a \neq c$ and $b \neq d$. In this case we should consider four subcases.

If $a<c$ and $b<d$, then $\Pi_{x-y} \subseteq\{2, p\}$ implies that each prime divisor of $2^{c-a} p^{d-b}-$ $\varepsilon / \delta$ is equal to 2 or $p$, which is not possible.

If $a<c$ and $b>d$, then $\Pi_{x-y} \subseteq\{2, p\}$ implies that $2^{c-a}-(\varepsilon / \delta) p^{b-d}=1$ and hence $\varepsilon=\delta$. In this case the Mihăilescu Theorem 4 ensures that $b-d=1$ and hence $2^{c-a}=p+1=2^{m}+2$ which is not possible (as $m>1$ ).

If $a>c$ and $b<d$, then $\Pi_{x-y} \subseteq\{2, p\}$ implies that $p^{d-b}-(\varepsilon / \delta) 2^{a-c}=1$, which implies that $\varepsilon=\delta$. The Mihăilescu Theorem 4 implies that $d-b=1$ and hence $2^{a-c}=$ $p-1=2^{m}$ and $a-c=m$. In this case $\{x, y\}=\left\{\varepsilon 2^{c+m-1} 2^{b}, \varepsilon 2^{c-1} p^{b+1}\right\}$.

The subcase $a>c$ and $b>d$ is forbidden by the inequality $2^{a-1} p^{b} \leq 2^{c-1} p^{d}$.

3. Assume that $p=2^{m}-1>3$ is Mersenne prime. In this case $m>2$ and $p$ is not Fermat. It is easy to check that every doubleton

$$
\begin{aligned}
&\{x, y\} \in\left\{\left\{\varepsilon 2^{a} p^{b}, \varepsilon 2^{a-1} p^{b}\right\},\left\{\varepsilon 2^{a-1} p^{b}, \varepsilon 2^{m+a-1} p^{b}\right\},\left\{\varepsilon 2^{a-1} p^{b+1}, \varepsilon 2^{m+a-1} p^{b}\right\},\right. \\
&\left.\left\{\varepsilon 2^{a-1} p^{b},-\varepsilon 2^{a-1} p^{b}\right\},\left\{\varepsilon 2^{a-1} p^{b},-\varepsilon 2^{a-1} p^{b+1}\right\}: a, b \in \mathbb{N}, \varepsilon \in\{-1,1\}\right\}
\end{aligned}
$$

has $A_{\{x, y\}}=\Pi_{x} \cup \Pi_{y} \cup \Pi_{x-y}=\{2, p\}$ and hence $\{x, y\} \in \mathcal{E}_{p}$.

Now assume that $\{x, y\} \in \mathcal{E}_{p}$ is an edge of the graph $\Gamma_{p}$. Then $\Pi_{x} \cup \Pi_{y} \cup \Pi_{x-y}=$ $A_{\{x, y\}}=\{2, p\}$ and $\{x, y\}$ can be written as $\left\{\varepsilon 2^{a-1} p^{b}, \delta 2^{c-1} p^{d}\right\}$ for some $a, b, c, d \in \mathbb{N}$, $\varepsilon, \delta \in\{-1,1\}$ with $2^{a-1} p^{b} \leq 2^{c-1} p^{d}$.

If $a=c, b=d$, then $\varepsilon=-\delta$ and $\{x, y\}=\left\{\varepsilon 2^{a-1} p^{b},-\varepsilon 2^{a-1} p^{b}\right\}$.

If $a=c$, then $b \leq d$ and the inclusion $\Pi_{x-y} \subseteq\{2, p\}$ implies that $\Pi_{p^{d-b}-\varepsilon / \delta} \subseteq\{2, p\}$ and hence $p^{d-b}-\varepsilon / \delta$ is a power of 2 . By the Mihăilescu Theorem $4, d-b \in\{0,1\}$. If $d-b=0$, then $\{x, y\}=\left\{\varepsilon 2^{a-1} p^{b},-\varepsilon 2^{a-1} p^{b}\right\}$ by the preceding case. So, we assume that $d-b=1$. If $\varepsilon=\delta$, then $p^{d-b}-\varepsilon / \delta=p-1=2^{m}-2$ is a power of 2 , which is not true as $m>2$. Therefore $\varepsilon=-\delta$ and $\{x, y\}$ is equal to $\left\{\varepsilon 2^{a-1} p^{b},-\varepsilon 2^{a-1} p^{b+1}\right\}$

If $b=d$, then $a \leq c$ and the inclusion $\Pi_{x-y} \subseteq\{2, p\}$ implies that $\Pi_{2^{c-a}-\varepsilon / \delta} \subseteq\{2, p\}$ and hence $2^{c-a}-\varepsilon / \delta$ is a power of $p$. By the Mihăilescu Theorem $4,2^{c-a}-\varepsilon / \delta \in\{1, p\}=$ $\left\{1,2^{m}-1\right\}$, which implies that $\varepsilon=\delta$ and $c-a \in\{1, m\}$. Therefore $\{x, y\}$ is equal to $\left\{\varepsilon 2^{a-1} p^{b}, \varepsilon 2^{a} p^{b}\right\}$ or $\left\{\varepsilon 2^{a-1} p^{b}, \varepsilon 2^{m+a-1} p^{b}\right\}$.

So, we assume that $a \neq c$ and $b \neq d$. By analogy with the case of Fermat primes, we can show that the subcases $(a<c$ and $b<d)$ and $(a>c$ and $b>d)$ are impossible. 


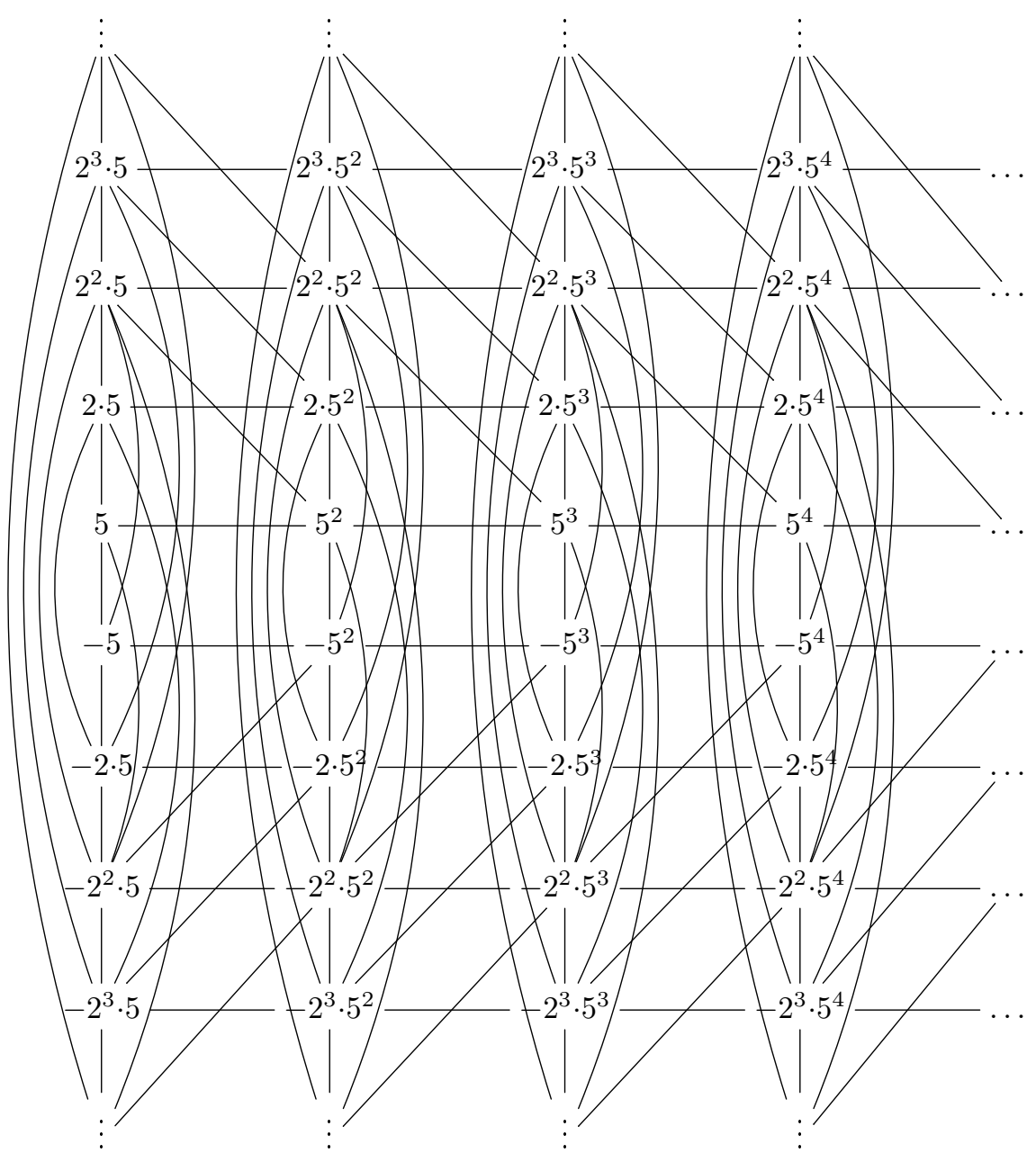

Figure 2. The graph $\Gamma_{5}$

If $a<c$ and $b>d$, then $\Pi_{x-y} \subseteq\{2, p\}$ implies that $2^{c-a}-(\varepsilon / \delta) p^{b-d}=1$, and hence $\varepsilon / \delta=1$. Then the Mihăilescu Theorem 4 ensures that $b-d=1$ and hence $2^{c-a}=p+1=2^{m}$ and $c-a=m$. In this case $\{x, y\}=\left\{\varepsilon 2^{a-1} p^{d+1}, \varepsilon 2^{a+m-1} p^{d}\right\}$.

If $a>c$ and $b<d$, then $\Pi_{x-y} \subseteq\{2, p\}$ implies that $p^{d-b}-(\varepsilon / \delta) 2^{a-c}=1$ and hence $\varepsilon / \delta=1$. Then Mihăilescu Theorem 4 implies that $d-b=1$ and hence $2^{a-c}=p-1=$ $2^{m}-2$, which is not possible as $m>2$.

4. Assume that $p$ is not Fermat-Mersenne. It is easy to check that every doubleton $\{x, y\} \in\left\{\left\{\varepsilon 2^{a-1} p^{b},-\varepsilon 2^{a-1} p^{b}\right\},\left\{\varepsilon 2^{a-1} p^{b}, \varepsilon 2^{a} p^{b}\right\}: a, b \in \mathbb{N}, \varepsilon \in\{-1,1\}\right\}$ 


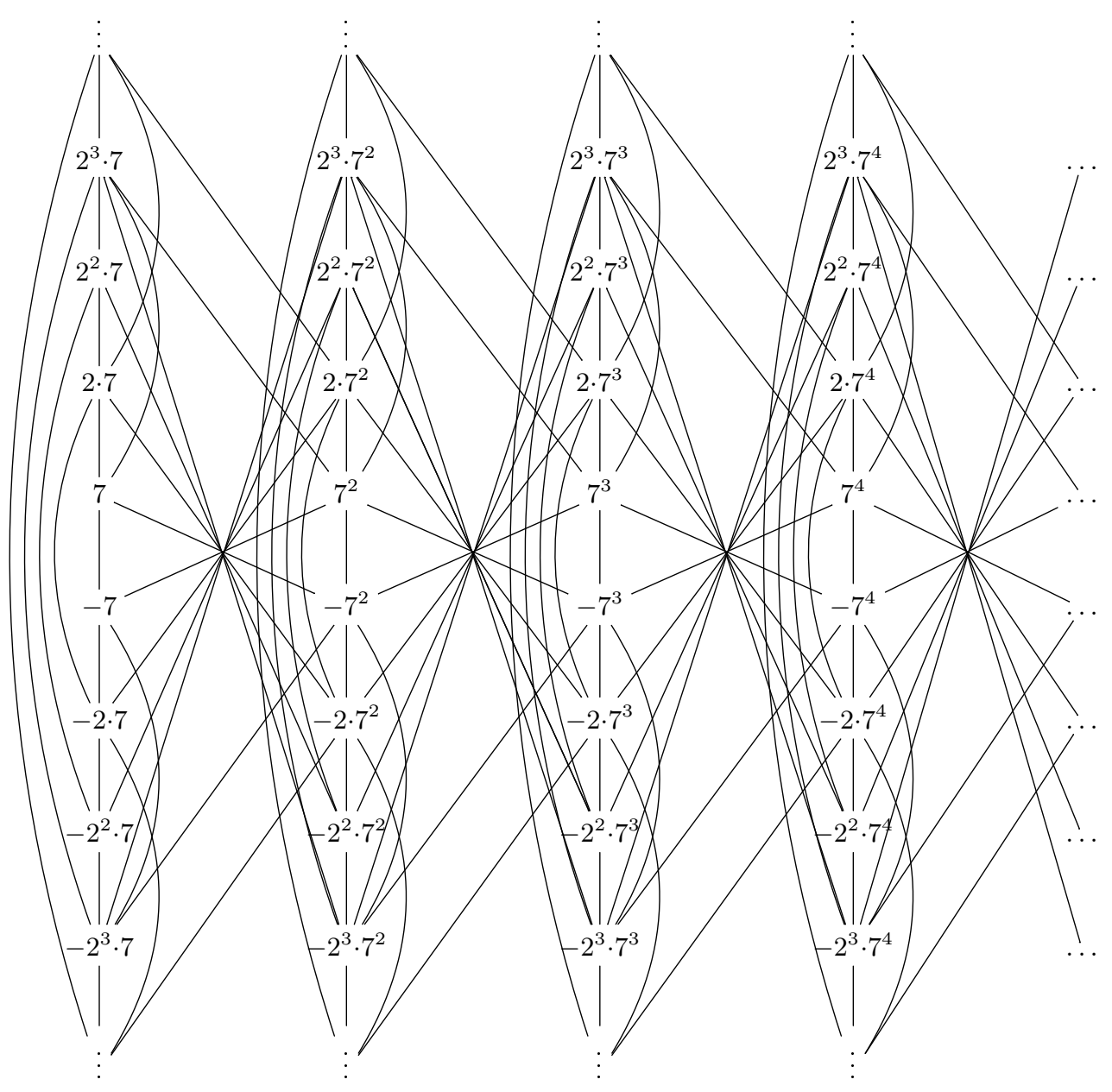

Figure 3. The graph $\Gamma_{7}$

has $A_{\{x, y\}}=\Pi_{x} \cup \Pi_{y} \cup \Pi_{x-y}=\{2, p\}$ and hence $\{x, y\} \in \mathcal{E}_{p}$.

Now assume that $\{x, y\} \in \mathcal{E}_{p}$ is an edge of the graph $\Gamma_{p}$. Then $\Pi_{x} \cup \Pi_{y} \cup \Pi_{x-y}=$ $A_{\{x, y\}}=\{2, p\}$ and $\{x, y\}$ can be written as $\left\{\varepsilon 2^{a-1} p^{b}, \delta 2^{c-1} p^{d}\right\}$ for some $a, b, c, d \in \mathbb{N}$, $\varepsilon, \delta \in\{-1,1\}$ with $2^{a-1} p^{b} \leq 2^{c-1} p^{d}$.

If $a=c$ and $b=d$, then $\varepsilon \neq \delta$ and $\{x, y\}=\left\{2^{a-1} p^{b},-2^{a-1} p^{b}\right\}$.

If $a=c$, then $b \leq d$ and the inclusion $\Pi_{x-y} \subseteq\{2, p\}$ implies that $\Pi_{p^{d-b}-\varepsilon / \delta} \subseteq\{2, p\}$ and hence $p^{d-b}-\varepsilon / \delta$ is a power of 2. By the Mihăilescu Theorem $4, d-b=1$ and hence $p$ is either Fermat prime or Mersenne prime which is not true.

If $b=d$, then $a \leq c$ and the inclusion $\Pi_{x-y} \subseteq\{2, p\}$ implies that $\Pi_{2^{c-a}-\varepsilon / \delta} \subseteq\{2, p\}$ and hence $2^{c-a}-\varepsilon / \delta$ is a power of $p$. By the Mihăilescu Theorem $4.2^{c-a}-\varepsilon / \delta \in\{1, p\}$. 

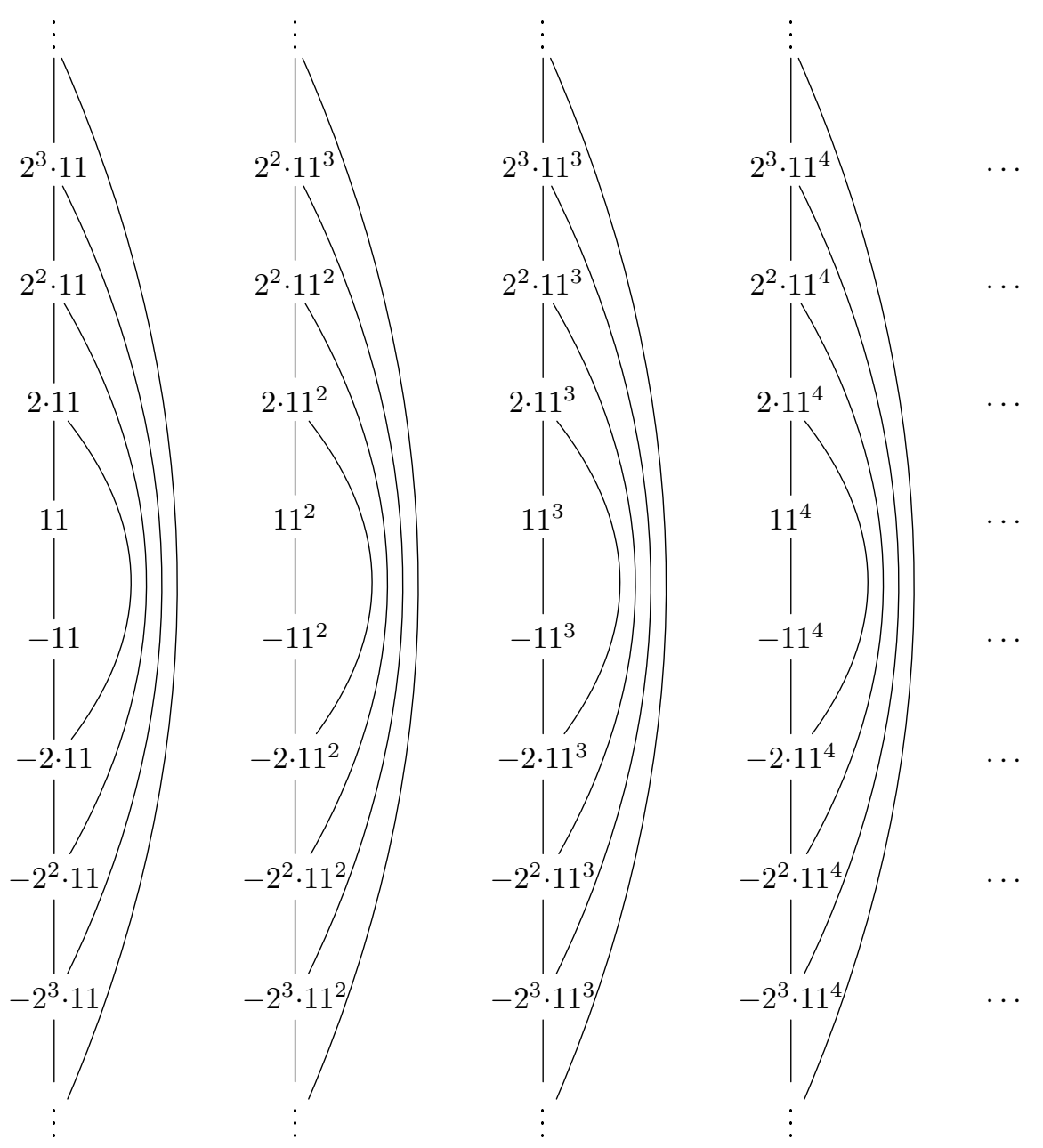

Figure 4. The graph $\Gamma_{11}$

Taking into account that $p$ is neither Fermat nor Mersenne prime, we conclude that if $\varepsilon=\delta, 2^{c-a}-1=1$ and hence $c-a=1$. Then $\{x, y\}=\left\{\varepsilon 2^{a-1} p^{b}, \varepsilon 2^{a} p^{b}\right\}$.

So, we assume that $a \neq c$ and $b \neq d$. By analogy with the case of Fermate primes, we can show that the subcases $(a<c$ and $b<d)$ and $(a>c$ and $b>d)$ are impossible.

If $a<c$ and $b>d$, then $\Pi_{x-y} \subseteq\{2, p\}$ implies that $2^{c-a}-(\varepsilon / \delta) p^{b-d}=1$. By the Mihăilescu Theorem $4 b-d=1$ and hence $p=2^{c-a}-1$ is a Mersenne prime, which is not true.

If $a>c$ and $b<d$, then $\Pi_{x-y} \subseteq\{2, p\}$ implies that $p^{d-b}-(\varepsilon / \delta) 2^{a-c}=1$. By the Mihăilescu Theorem $4 d-b=1$ and hence $p=1+2^{a-c}$ is a Fermat prime, which is not true. 
In Figures $1,2,3,4$ we draw the graphs $\Gamma_{p}$ for $p$ equal to $3,5,7,11$. Observe that 3 is both Fermat and Mersenne prime, 5 is Fermat prime, 7 is Mersenne prime and 11 is not Fermat-Mersenne.

Lemma 20. Let $p$ be an odd prime number and $h$ be a positive homeomorphism of the Kirch space.

(1) If $p$ is Fermat-Mersenne, then $h(p)=p$;

(2) If $p$ is not Fermat-Mersenne, then $h(p)=p^{n}$ for some $n \in \mathbb{N}$.

Proof. 1. Lemma 19(1) implies that the degree of \pm 3 in the graph $\Gamma_{3}$ is equal to 8 but the other vertices have degree at least 9 . Hence $h(3)= \pm 3$. Assume that $h(3)=-3$. Then by Lemma 14 and by Lemma 13

$$
\{2,3\}=A_{\{2,3\}}=A_{h(\{2,3\})}=A_{\{2,-3\}}=\{2,3,5\}
$$

but this is not true and hence $h(3)=3$.

Assume that $p>3$ is either Fermat or Mersenne prime. Lemma $19(2,3)$ implies that the degree of $\pm p$ in the graph $\Gamma_{p}$ is 4 but the other vertices have degree at least 5 . Hence $h(p)= \pm p$. Assume that $h(p)=-p$. By Lemma 14. $A_{\{1, p\}}=A_{\{1, h(p)\}}=A_{\{1,-p\}}$, so $\{p\} \cup \Pi_{p-1}=\{p\} \cup \Pi_{p+1}$, according to Lemma 2 . This implies that $\Pi_{p-1}=\Pi_{p+1}=\{2\}$. Hence $p$ is both Fermate and Mersenne which is possible iff $p=3$ and this contradicts our assumption. Therefore $h(p)=p$.

2. Let $p$ be an odd prime number, which is not Fermat-Mersenne. Lemma $19(4)$ implies that the set $\pm p^{\mathbb{N}}=\left\{\varepsilon p^{n}: n \in \mathbb{N}, \varepsilon \in\{-1,1\}\right\}$ coincides with the set of vertices of order 2 in the graph $\Gamma_{p}$. Taking into account that $h \uparrow V_{p}$ is an isomorphism of the graph $\Gamma_{p}$, we conclude that $h(p)= \pm p^{n}$ for some $n \in \mathbb{N}$. Assume that $h(p)=-p^{n}$. Then $h(\{-1, p\})=\left\{-1,-p^{n}\right\}$. By Lemma 13, $A_{\{-1, p\}}=A_{\left\{-1,-p^{n}\right\}}$, so $\{p\} \cup \Pi_{p+1}=$ $\{p\} \cup \Pi_{p^{n}-1}$, according to Lemma 2 . Since $\{p\}$ does not intersect $\Pi_{p+1}$ and $\Pi_{p^{n}-1}$ we conclude that $\Pi_{p+1}=\Pi_{p^{n}-1}$. Hence we get the inclusion $\Pi_{p-1} \subseteq \Pi_{p^{n}-1}=\Pi_{p+1}$. If some prime number $d$ divides $p-1$ then the inclusion $\Pi_{p-1} \subseteq \Pi_{p+1}$ implies that $d$ divides $p+1$, consequently $d$ divides the difference $(p+1)-(p-1)=2$ and hence $d=2$. As a consequence, $\Pi_{p-1}=\{2\}$ and $p-1=2^{m}$ for some $m \in \mathbb{N}$ which contradicts the assumption that $p$ is not Fermat prime. Hence $h(p)=p^{n}$.

Lemma 21. For any positive homeomorphism $h$ of the Kirch space and any prime number $p$ we have $h(p)=p$.

Proof. If $p=2$, then $h(p)=p$ by Lemma 14. If $p$ is Fermat-Mersenne, then $h(p)=p$ by Lemma 20. So, we assume $p$ is not Fermat-Mersenne. By Lemma 20, $h(p)=p^{n}$ for some $n \in \mathbb{N}$. By Lemmas 2 , 14 and 13 .

$$
\{p\} \cup \Pi_{p-1}=A_{\{1, p\}}=A_{\{1, h(p)\}}=A_{\left\{1, p^{n}\right\}}=\{p\} \cup \Pi_{p^{n}-1}
$$

and hence $\Pi_{p^{n}-1}=\Pi_{p-1}$. Since $p$ is not Mersenne prime, Zsigmondy Theorem 5 guarantees that $n=1$ and hence $h(p)=p^{1}=p$.

Lemma 22. The positive homeomorphism group of the Kirch space is trivial.

Proof. To derive a contradiction, assume that the Kirch space admits a homeomorphism $h$ such that $h(x) \neq x$ for some number $x$. By the Hausdorff property of the Kirch space and the continuity of $h$, there exists a neighborhood $O_{x}$ of $x$ in the Kirch topology such 
that $h\left[O_{x}\right] \cap O_{x}=\varnothing$. By the definition of the Kirch topology, there exists a square-free number $b$ such that $\Pi_{b} \cap \Pi_{x}=\varnothing$ and $x+b \mathbb{Z} \subseteq O_{x}$. By the Dirichlet Theorem 3 , the arithmetic progression $x+b \mathbb{N} \subseteq x+b \mathbb{Z}$ contains some prime number $p$. Then $h\left[O_{x}\right] \cap O_{x}=$ $\varnothing$ implies $h(p) \neq p$, which contradicts Lemma 21 .

Our final lemma completes the proof of Theorem 1

Lemma 23. Any homeomorphism $h$ of the Kirch space $\mathbb{Z} \bullet$ is equal to $i: \mathbb{Z} \rightarrow \mathbb{Z}$, $i: x \mapsto x$ or to $j: \mathbb{Z} \bullet \rightarrow \mathbb{Z} \bullet j: x \mapsto-x$.

Proof. If $h$ is positive, then $h=i$ by previous Lemma. If $h$ is not positive then $h(1)<0$ and $j \circ h(1)>0$. Then the homeomorphism $j \circ h$ is positive and equals $i$ by the preceding case. This implies that

$$
h=i \circ h=(j \circ j) \circ h=j \circ(j \circ h)=j \circ i=j .
$$

\section{ACKNOWLedgement}

The author expresses her sincerely thanks to Taras Banakh for his generous help during preparation of this paper.

\section{REFERENCES}

1. T. Apostol, Introduction to analytic number theory, Springer-Verlag, New York, 1976.

2. T. Banakh, D. Spirito, and S. Turek, The Golomb space is topologically rigid, Comment Math. Univ. Carolin. (accepted); arXiv:1912.01994, 2019, preprint.

3. T. Banakh, Y. Stelmakh, and S. Turek, The Kirch space is topologically rigid, Topology Appl. (accepted); arXiv:2006.12357, 2020, preprint.

4. P. Dirichlet, Lectures on number theory. Supplements by R. Dedekind. Translated from the 1863 German original and with an introduction by John Stillwell, Amer. Math. Soc., Providence, RI; London Math. Soc., London, 1999.

5. G. A. Jones and J. M. Jones, Elementary number theory, Springer, 2012.

6. A. M. Kirch, A countable, connected, locally connected Hausdorff space, Amer. Math. Monthly 76 (1969), no. 2, 169-171. DOI: $10.2307 / 2317265$

7. T. Metsänkylä, Catalan's conjecture: another old Diophantine problem solved, Bull. Amer. Math. Soc. (N.S.) 41 (2004), no. 1, 43-57. DOI: 10.1090/S0273-0979-03-00993-5

8. P. Mihăilescu, Primary cyclotomic units and a proof of Catalan's conjecture, J. Reine Angew. Math. 572 (2004), 167-195. DOI: 10.1515/crll.2004.048

9. N. J. A. Sloane, The on-line encyclopedia of integer sequences, (https://oeis.org).

10. M. Roitman, On Zsigmondy primes, Proc. Amer. Math. Soc. 125 (1997), no. 7, 1913-1919. DOI: $10.1090 /$ S0002-9939-97-03981-6

11. D. Spirito, The Golomb topology on a Dedekind domain and the group of units of its quotients, Topology Appl. 273 (2020), 107101. DOI: 10.1016/j.topol.2020.107101

12. K. Zsigmondy, Zur Theorie der Potenzreste, Monatsh. Math. Phys. 3 (1892), 265-284. DOI: $10.1007 /$ BF01692444 
HOMEOMORPHISMS OF THE SPACE OF NONZERO INTEGERS

ISSN 2078-3744. Вісник Львів. ун-ту. Серія мех.-мат. 2020. Випуск 89

\title{
ГОМЕОМОРФІЗМИ ПРОСТОРУ НЕНУЛЬОВИХ ЦІЛИХ ЧИСЕЛ 3 ТОПОЛОГІЕЮ КІРХА
}

\author{
Ярина СТЕЛЬМАХ \\ Лъвівсъкий націоналъний університет імені Івана Франка, \\ вул. Університетсъка, 1, 79000, Лъвів \\ e-mails: yarynziya@ukr.net
}

Топологія Голомба (відповідно Кірха) на множині $\mathbb{Z} \bullet$ ненульових цілих чисел породжується базою, що складається з арифметичних прогресій $a+b \mathbb{Z}=\{a+b n: n \in \mathbb{Z}\}$, де $a \in \mathbb{Z} \bullet$ i $b$ - взаємно просте з $a$ число, (що не ділиться на квадрат жодного простого числа). У 2019 році Даріо Спіріто довів, що простір ненульових цілих чисел з топологією Голомба допускає лише два автогомеоморфізми. Ми доводимо аналогічний факт для простору ненульових цілих, наділеного топологією Кірха: він також має рівно два автогомеоморфізми.

Ключові слова: топологія Кірха, суперзв'язний простір, суперзв'язуюча частково впорядкована множина. 